\title{
Chemotherapy-induced intestinal inflammatory responses are mediated by exosome secretion of double-strand DNA via AIM2 inflammasome activation
}

Qiaoshi Lian ${ }^{1,4, *}$, Jun $\mathrm{Xu}^{2,4, *}$, Shanshan Yan $^{1,5,{ }^{*}}$, Min Huang ${ }^{2, *}$, Honghua Ding ${ }^{6}$, Xiaoyu $\mathrm{Sun}^{3}$, Aiwei $\mathrm{Bi}^{2}$, Jian Ding ${ }^{2}$, Bing Sun ${ }^{1,3}$, Meiyu Geng ${ }^{2}$

${ }^{I}$ State Key Laboratory of Cell Biology, CAS Center for Excellence in Molecular Cell Science, Shanghai Institute of Biochemistry and Cell Biology, Chinese Academy of Sciences; University of Chinese Academy of Sciences, 320 Yueyang Road, Shanghai 200031, China; ${ }^{2}$ Division of Antitumor Pharmacology, State Key Laboratory of Drug Research, Shanghai Institute of Materia Medica, Chinese Academy of Sciences, Shanghai 201203, China; ${ }^{3}$ CAS Key Laboratory of Molecular Virology \& Immunology, Institute Pasteur of Shanghai, Chinese Academy of Sciences, 320 Yueyang Road, Shanghai 200031, China; ${ }^{4}$ University of Chinese Academy of Sciences, Beijing 100049, China; ${ }^{5}$ School of Life Sciences, University of Science and Technology of China, Hefei 230022, China; ${ }^{6}$ Department of Oncology, Shanghai General Hospital, Shanghai Jiao Tong University School of Medicine, Shanghai 200080, China

Chemotherapies are known often to induce severe gastrointestinal tract toxicity but the underlying mechanism remains unclear. This study considers the widely applied cytotoxic agent irinotecan (CPT-11) as a representative agent and demonstrates that treatment induces massive release of double-strand DNA from the intestine that accounts for the dose-limiting intestinal toxicity of the compound. Specifically, "self-DNA" released through exosome secretion enters the cytosol of innate immune cells and activates the AIM2 (absent in melanoma 2) inflammasome. This leads to mature IL-1 $\beta$ and IL-18 secretion and induces intestinal mucositis and late-onset diarrhoea. Interestingly, abrogation of AIM2 signalling, either in AIM2-deficient mice or by a pharmacological inhibitor such as thalidomide, significantly reduces the incidence of drug-induced diarrhoea without affecting the anticancer efficacy of CPT-11. These findings provide mechanistic insights into how chemotherapy triggers innate immune responses causing intestinal toxicity, and reveal new chemotherapy regimens that maintain anti-tumour effects but circumvent the associated adverse inflammatory response.

Keywords: dsDNA; AIM2; exosome; inflammasome; chemotherapy; intestinal toxicity

Cell Research (2017) 27:784-800. doi:10.1038/cr.2017.54; published online 14 April 2017

\section{Introduction}

Chemotherapies, which remain the primary treatment choice for many types of advanced cancers, lead to cytotoxic anti-tumour activity via a variety of mechanisms. In addition to the direct killing of cancer cells, the cy-

\footnotetext{
*These four authors contributed equally to this work.

Correspondence: Meiyu Geng ${ }^{\mathrm{a}}$, Bing Sun ${ }^{\mathrm{b}}$, Jian Ding ${ }^{\mathrm{c}}$, Min Huang ${ }^{\mathrm{d}}$

${ }^{a}$ E-mail: mygeng@simm.ac.cn

bE-mail: bsun@sibs.ac.cn

${ }^{c}$ E-mail: jding@simm.ac.cn

dE-mail: mhuang@simm.ac.cn

Received 8 November 2016; revised 19 February 2017; accepted 28 February 2017; published online 14 April 2017
}

totoxic effects of chemotherapies are often associated with potent immunostimulatory effects. These include pro-inflammatory cytokine secretion [1], myeloid cell activation and recruitment [1], and T-cell activation [2, $3]$, all of which significantly contribute to the anti-tumour efficacy of chemotherapy-based cancer treatments [4]. Indeed, accumulating evidence indicates that the therapeutic efficacy of many available chemotherapeutics relies on their capacity to elicit anti-tumour immune responses. Although approaches enhancing chemotherapy-induced anti-tumour immune responses are nowadays preferred, excessive immune responses could also trigger an uncontrolled toxicity of chemotherapy that is largely ignored.

Immunogenic responses induced by cytotoxic agents 
rely on exposure to molecules released from dying tumour cells that function either as adjuvant or antigen signals for the innate immune system [5-7]. These signals, such as the high mobility group protein B1, released during cellular necrosis, together with uric acid and ATP are known as damage-associated molecular patterns (DAMPs) [7, 8]. Self-DNA, a key cause of inflammatory and autoimmune disease, is an important DAMP released during chemotherapy [9-11]. Cytotoxic anticancer agents, such as cisplatin, etoposide, or radiation therapy, have been shown to trigger innate immune responses that involve the leakage of nuclear-derived self-DNA from tumour cells [12-14]. The released self-DNA is able to access the cytosol of dendritic cells to activate stimulator of interferon gene-dependent cytokine production via recognition by the cytosolic DNA sensor cGAS (cyclic GMP-AMP synthase) [15]. To date, the precise origins and mechanistic details of self-DNA-mediated systemic or local immunogenic responses remain unclear. Whether, in addition to anti-tumour immunity, massive self-DNA release could lead to broader immunogenic responses, in particular chemotherapy-associated adverse effects, has long been ignored. Gaining mechanistic insights into these immunogenic responses may be essential to maximize the clinical benefits of chemotherapy agents.

Gastrointestinal syndrome is a well-recognized side effect associated with a variety of chemotherapeutic agents, particularly irinotecan (CPT-11) and fluorouracil (5FU). As the first-line treatment for colorectal cancer (CRC), the clinical benefit of CPT-11 is limited by its adverse effect of severe diarrhoea, which occurs in $\sim 40 \%$ of patients receiving CPT-11 treatment [16-18]. Persistent or severe diarrhoea is not only a life-threatening side effect for CRC patients, but also can influence efficiency of chemotherapy through a need to reduce treatment doses or discontinue therapy. Thus far, the mechanism of CPT-11-triggered life-threatening gastrointestinal syndrome is poorly understood. Clinical management of diarrhoea reflects the need for recognition of the early warning signs and the need for early and aggressive management [19]. Of note, several lines of evidence suggest that CPT-11-triggered diarrhoea is associated with the production of the pro-inflammatory cytokines IL-1 $\beta$ and IL-18 [20], suggesting an immunogenic response. Previous studies have also demonstrated an anticancer effect of CPT-11-associated anti-tumour immunity [21]. Consequently, we consider CPT-11-induced diarrhoea as an ideal model to dissect the mechanism of the interplay between immunogenic response-associated anti-tumour efficacy and the adverse effects of chemotherapy. This study may facilitate the design of a new strategy for the clinical use of chemotherapeutic agents that are able to maintain the balance between anti-tumour effects and inflammatory response associated toxicities.

\section{Results}

Double-strand DNA release is associated with CPT-11 treatment-induced diarrhoea

Evidence suggests that CPT-11-induced diarrhoea is an inflammation-mediated side effect, but the immunogen responsible remains unclear [20, 22-24]. To investigate whether CPT-11-induced diarrhoea was associated with the release of double-strand DNA (dsDNA), we first analysed clinical samples from CRC patients who received a CPT-11-based chemo-regimen treatment. Among a total of 69 advanced CRC patients, 40\% exhibited grade 1-3 diarrhoea (Supplementary information, Figure S1A), an incidence similar to previous reports [16-18]. Assessment of the dsDNA concentration in patient serum was performed by quantitative staining with PicoGreen, a dye specific to dsDNA and insensitive to single-strand DNA or RNA. By comparing the total dsDNA concentration before and after CPT-11 treatment, we noticed that the dsDNA concentration in the serum of patients, particularly those with grade 2-3 diarrhoea, was elevated $48 \mathrm{~h}$ following CPT-11 administration (Figure 1A-1B). Interestingly, the subset of patients with higher-grade diarrhoea showed higher levels of serum dsDNA than the remaining patients (Figure 1C), suggesting that the higher dsDNA concentration was associated with CPT-11-induced severe diarrhoea.

To test this hypothesis and to identify a detailed mechanism, we decided to attempt to mirror this clinical observation with an intestinal mucositis mouse model of CPT-11-associated diarrhoea [25]. The model was generated in $\mathrm{C} 57 \mathrm{BL} / 6$ mice bearing or non-bearing murine colon carcinoma MC38 xenografts. The mucositis severity was assessed by scoring survival rate, severity of diarrhoea, intestine length, and histopathology. In both tumour-bearing and non-tumour-bearing mice, successive intraperitoneal injections of CPT-11 for 4 consecutive days led to severe intestinal inflammation resulting in shortening of the small intestine but not the colon (Figure 1D-1E). The results were confirmed by histopathological changes in the small intestine (Figure 1F; Supplementary information, Figure S1B-S1C), largely reflecting the pathological features of CPT-11-induced severe diarrhoea seen in clinical patients [16].

Interestingly, large quantities of free dsDNA were detected in the peritoneal lavage fluid in both non-tumour-bearing mice and tumour-bearing mice (Figure $1 \mathrm{G}$ ) during induction of mucositis (5 days post CPT- 

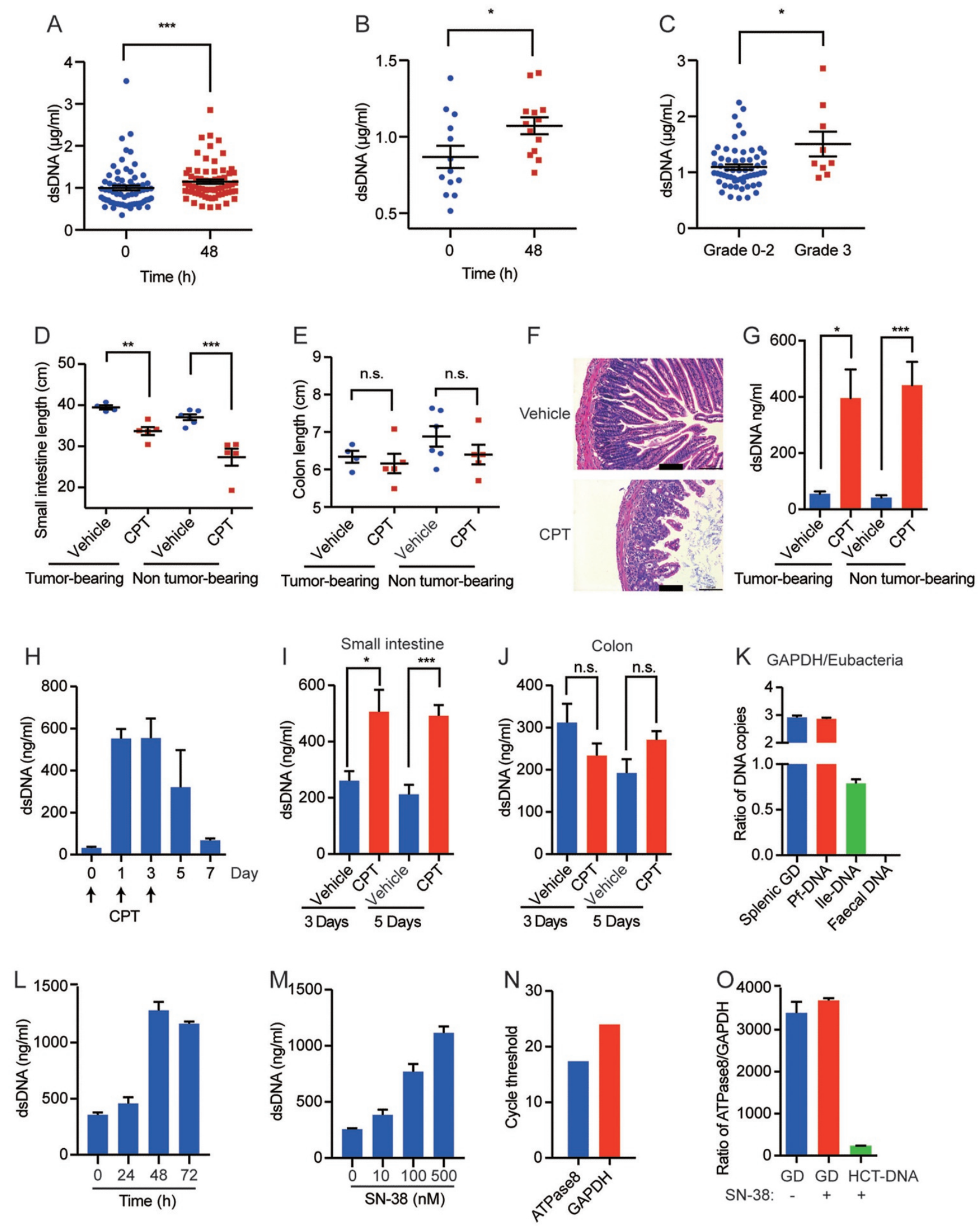
11 injection). The dsDNA concentration peaked on day 1-3 and then declined to a lower level following CPT11 treatment (Figure 1H). The kinetic change in dsDNA concentration mimicked the clinical course observed in the patients. Consistent with the severe damage in the small intestinal tract (Figure 1F), we observed greater dissociative dsDNA accumulation in the fluid flushed from the small intestine (Figure 1I). In contrast, no dsDNA induction was observed in the fluid flushed from the colon (Figure 1J). Further quantitative PCR (qPCR) analysis confirmed that the accumulated dsDNA was mainly derived from host cells and not of intestinal bacterial origin by comparison with the positive control (genomic DNA from mouse splenocytes) and negative control (total DNA from mouse faeces) (Figure 1K). These data suggest that there is a close association between CPT-11-induced intestinal mucositis and dsDNA production. CPT11 treatment may trigger the host cell to release massive amounts of self-dsDNA, which serves as a strong immune stimulant to initiate the development of mucositis.

\section{CPT-11 directly triggers nuclear genomic DNA release from proliferating cells}

Next, we examined dsDNA release induced by CPT11 treatment, which had not been clearly described in previous investigations of cytotoxic agents or radiation-induced dsDNA leakage $[13-15,26]$. CPT-11 is known to specifically target proliferating cells, such as tumour cells and intestinal epithelial cells [27]. It was also noted that dsDNA amount in tumour-bearing and non-bearing mice was comparable, suggesting that intestinal epithelial cells, and not tumour cells, might be the major source of dsDNA (Figure 1G). We hence chose transformed HCT-116 cells as an intestinal epithelial cell line model [28-31] to assess whether CPT-11 treatment could directly trigger self-DNA release in vitro. HCT116 cells were treated with $\mathrm{SN}-38$, an active form of the pro-drug CPT-11. Similar to the results obtained in vivo, dsDNA was detected in the culture medium of HCT-116 cells following SN-38 treatment (Figure 1L). In addition, SN-38 triggered dsDNA release from HCT-116 cells in a time- and dose-dependent manner (Figure 1L-1M). We also tested another DNA-damaging agent, cisplatin, and observed a similar pattern of dsDNA release in HCT-116 cells (Supplementary information, Figure S1D).

Released dsDNA could originate from either nuclear genomic DNA or mitochondrial DNA (mtDNA) [32]. To identify the origin of the released dsDNA, we examined the levels of the genes for ATPase8 (a mtDNA marker) and GAPDH (a marker for nucleic genomic DNA) by qPCR in the dsDNA released from HCT-116 cells (HCTDNA). Indeed, both ATPase8 and GAPDH genes were detected in the dsDNA, suggesting that both nuclear genomic DNA and mtDNA contributed to the released dsDNA in the cell culture medium (Figure $1 \mathrm{~N}$ ). We also observed that the ATPase8/GAPDH gene ratio in HCTDNA was much lower than in DNA extracted from total cells (Figure 1O). Given the large copy number of mtDNA in cells, this suggested that nucleic genomic DNA makes the predominant contribution to CPT-11-released dsDNA. Taken together, these data indicate that CPT11 directly triggers the release of the nuclear genomic in proliferating cells.

Self-DNA induced by CPT-11 promotes IL- $1 \beta$ and IL-18 maturation in an AIM2-dependent manner

The consequences of high amounts of dsDNA in vivo remain unclear. We suspected that the dsDNA release

Figure 1 CPT-11 triggers dsDNA release in vivo and in vitro. (A-C) Concentration of dsDNA in patient plasma before or at $48 \mathrm{~h}$ post CPT-11 (CPT) treatment. Significance determined using paired or unpaired $t$-test. (A) Total patients; (B) patients with grade 2-3 diarrhoea; (C) patients with grade 0-2 diarrhoea versus those with grade 3 at $48 \mathrm{~h}$ after treatment. (D-G) Tumour-bearing or non-tumour-bearing C57BL/6 mice were treated (i.p.) with CPT-11 (75 mg/kg for tumour-bearing mice, 90 $\mathrm{mg} / \mathrm{kg}$ for non-tumour-bearing mice) daily for 4 consecutive days and were sacrificed on day 5. (D) Length of small intestines; (E) length of colons; (F) representative images of H\&E histopathology of ileum sections from non-tumour-bearing mice; (G) concentration of dsDNA in cell-depleted peritoneal lavage fluid. (H-J) Time-dependent release of dsDNA in cell-depleted Pf (H), flushed fluid of ileum (I), or flushed fluid of colon (J). Non-tumour-bearing C57BL/6 mice were sacrificed at indicated days following CPT-11 treatment. Arrows indicate CPT-11 administration (90 mg/kg, i.p.) on day 0-3. (K) Quantitative PCR analysis of host GAPDH and total bacterial (Eubacteria) DNA copies in DNA species isolated from Pf-DNA and ileum fluid (Ile-DNA). C57BL/6 mice were administrated with CPT-11 (90 mg/kg, i.p.) daily for 4 consecutive days and were sacrificed on day 5 . Splenic GD (genomic DNA from mouse splenocytes) and faecal DNA (DNA from mouse faeces) were used as controls. (LM) DNA concentrations in the culture medium of HCT-116 cells treated with $500 \mathrm{nM} \mathrm{SN-38}$ at the indicated times (L) or with the indicated concentrations at $72 \mathrm{~h}(\mathrm{M})$. (N-O) Quantitative PCR analysis of ATPase8 and GAPDH DNA copies in the DNA released from HCT-116 cells $(\mathrm{N})$ or in GD from HCT-116 cells (O). HCT-116 cells were treated with SN-38 (500 nM) for $72 \mathrm{~h}$ before harvest for DNA extraction. GD from HCT-116 cells; HCT-DNA, DNA released from HCT-116 cells. Each symbol represents one patient (A, B, and $\mathbf{C}$ ) or one mouse (D, E). The data (D-O) are representative of three independent experiments and depict means \pm SEM. ${ }^{*} P<0.05$, ${ }^{* *} P<0.01,{ }^{* * *} P<0.001$. GD, genomic DNA; NS, not significant; Pf, peritoneal fluid. 
may be related to CPT-11-induced intestinal inflammation. Hence, we measured IL- $1 \beta$ production in a CPT-11induced mouse mucositis model. Upon CPT-11 administration, the pattern of IL- $1 \beta$ secretion in the peritoneal lavage fluid displayed similar kinetics to that of the dsDNA level (Figures 2A, 1H). This finding suggested that the dsDNA released from intestinal epithelial cells accumulates in the intestinal microenvironment, and in turn triggers inflammasome activation to produce IL-1 $\beta$. To test this possibility, the released self-DNA was isolated from SN-38-treated HCT-116 cell cultures or from the ileum of CPT-11-treated non-tumour-bearing mice and was then transfected into bone marrow-derived macrophages (BMMs). The released self-DNA dramatically induced mature IL-1 $\beta$ secretion in both lipopolysaccharide (LPS)-primed (Figure 2B) and non-LPS-primed (Figure 2C) BMMs, using murine genomic DNA as a positive control. We further assessed the level of the p10 subunit of caspase 1 to evaluate inflammasome activation. Indeed, self-DNA released from both intestinal cell culture and intestinal tissues markedly triggered caspase 1 activation (Figure 2D).

AIM2 is a cytosolic dsDNA receptor in the inflammasome pathway that mediates the cleavage of inactive precursor IL-1 $\beta$ and IL-18 into mature cytokines [3335]. AIM2-deficient mice exhibit a defective IL-1ß/IL-18 maturation response to dsDNA stimulation and are more sensitive to infection by pathogens, such as Francisella tularensis and vaccinia virus [36, 37]. Therefore, we investigated whether IL-1 $\beta$ production and inflammasome activation stimulated by released dsDNA was mediated by AIM2. To test this hypothesis, released self-DNA isolated from SN-38-treated HCT-116 cell cultures or from the ileum of CPT-11-treated mice was transfected into LPS-primed BMMs or bone-marrow-derived dendritic cells (BMDCs) from wild-type- or AIM2-deficient mice. The data showed that the self-DNA-induced production of IL-1 $\beta$ and IL-18 was almost completely abolished in BMMs from AIM2-deficient mice compared to the wildtype (Figure 2E-2F). We confirmed the deficiency of AIM2-deficient mice by analysing AIM2 mRNA levels (Supplementary information, Figure S2A) and a functional assay (Supplementary information, Figure S2BS2C). By contrast, the secretion of IL-6, which depends on toll-like receptor signalling, was not affected by AIM2 deficiency (Figure 2G). BMDCs transfected with DNAs of various origins displayed similar phenotypes (Figure 2H-2I).

To further verify that IL- $1 \beta$ production was a DNA-dependent function, DNase and RNase were introduced to digest DNA and RNA, respectively. As expected, treatment with DNase abolished nucleic acid-stimulated IL-
$1 \beta$ secretion, whereas RNase pretreatment had a minimal effect (Figure 2J). We also measured IL-6 production in response to DNase or RNase treatment and did not observe an effect (Figure 2K). These data indicate that IL-1 $1 \beta / I L-18$ cytokine production is a specific event stimulated by released self-DNA in an AIM2-dependent manner.

Exosomes deliver released self-DNA into the cytosol of innate immune cells

AIM2 is a cytosolic DNA sensor that can only be activated by dsDNA in the cytosol, regardless of whether it is derived from pathogens, damaged tissue, or delivered by cationic liposomes [38]. This observation raises the question of whether dsDNA resulted from CPT-11 induction can enter the cytosol. To address this question, we utilized an in vitro co-culture system composed of HCT-116 cells and murine innate immune cells, namely primary BMMs and BMDCs. HCT-116 cells in which genomic DNA was visualized by the DNA-intercalating dye DRAQ5 were stimulated by SN-38 treatment followed by co-culturing with macrophages or dendritic cells for $24 \mathrm{~h}$. The macrophages and dendritic cells were then selected by flow cytometry based upon staining to reveal murine $\mathrm{CD} 11 \mathrm{~b}$ or $\mathrm{CD} 11 \mathrm{c}$ staining, respectively. Approximately $40 \%$ of the $\mathrm{CD} 11 \mathrm{~b}^{+}$cells (Figure $3 \mathrm{~A}$ ) and $44 \%$ of the $\mathrm{CD} 11 \mathrm{c}^{+}$cells (Supplementary information, Figure S3A) were positive for HCT-116-derived DRAQ5 staining in contrast to controls, suggesting that HCT-116derived DNA was captured by innate immune cells.

It remains unclear how dsDNA enters macrophages and dendritic cells. Membrane extracellular vesicles, such as microparticles and exosomes, are known as vital transporters of intracellular signals to the outside environment, serving as mediators of intercellular communication $[39,40]$ and tumour-derived immune cell activation has been shown to be mediated by exosomes and microparticles $[41,42]$. Thus, we speculated that extracellular vesicles were likely involved in CPT-11triggered self-DNA release. By separating the particle fractions from SN-38-treated cell culture supernatants using gradient centrifugation [42, 43] (Figure 3B), we discovered that most dsDNA in HCT-116 culture medium was likely present in the exosomes (Figure 3C). This was supported by the size distribution of isolated particles, most of which were under $50 \mathrm{~nm}$ (Figure 3D) and relatively smaller than typical microparticles [43, 44]. Exosome secretion was also confirmed by immunoblotting of CD63, a molecular marker for exosomes (Figure 3E). We also tested exosome presence in vivo following CPT-11 treatment of mice. We found a remarkable increase in the CD63 expression level in the villi of the small intestine 
A

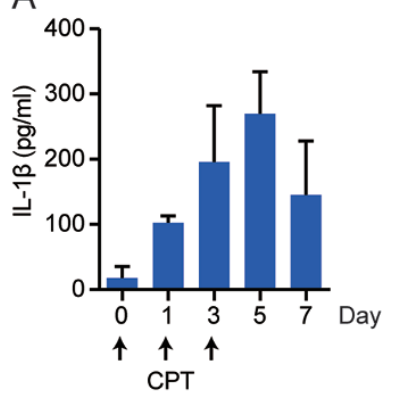

E

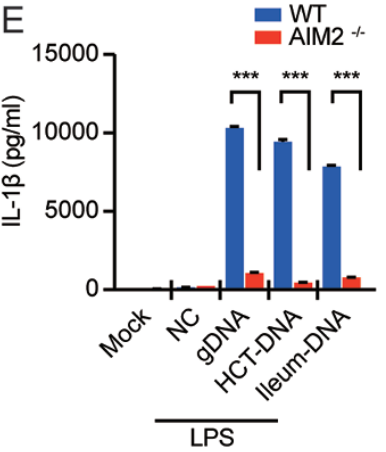

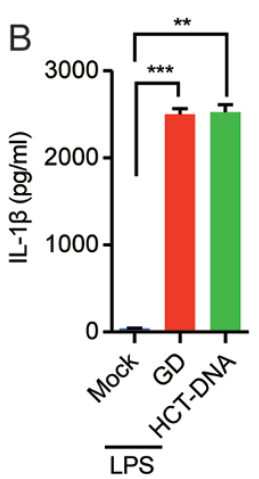

F

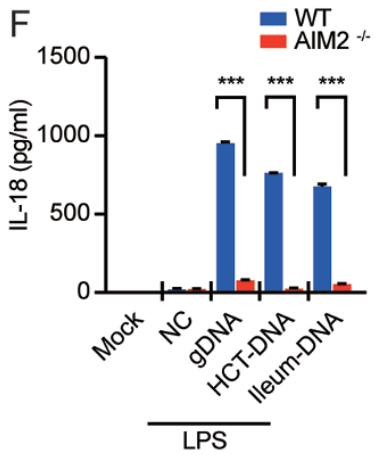

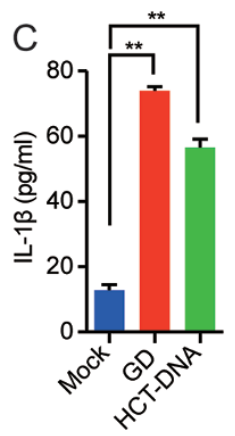

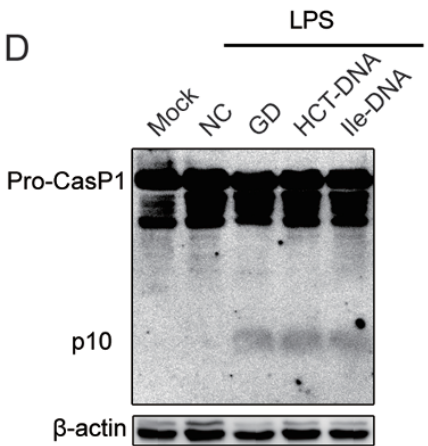

G
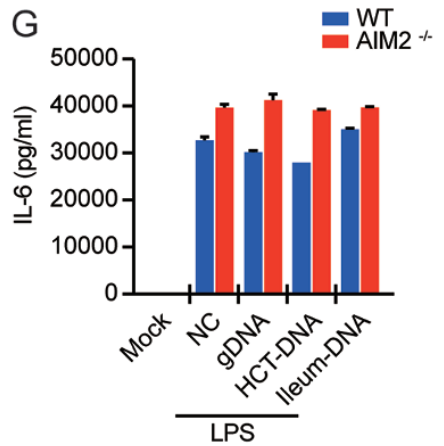
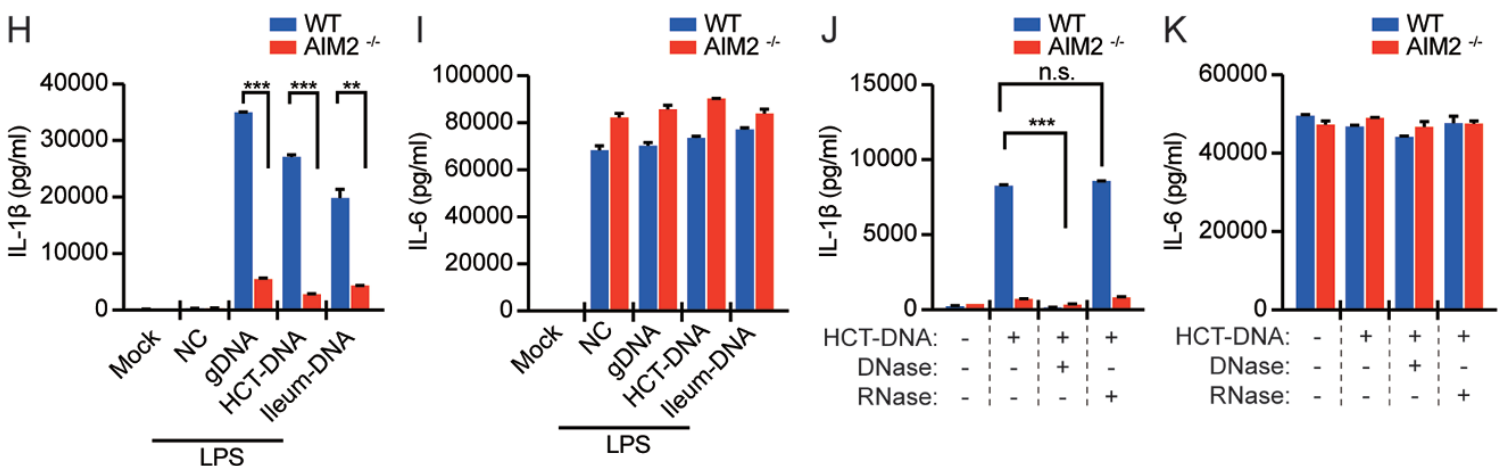

Figure 2 CPT-11-induced dsDNA release activates the AIM2 inflammasome. (A) Time-dependent production of IL-1 $\beta$ in the cell-depleted peritoneal fluid. C57BL/6 mice were sacrificed at indicated days following CPT-11 treatment. Arrows indicated CPT-11 administration (90 mg/kg, i.p.) from day 0 to 3. (B-C) Production of IL-1 $\beta$ in BMMs transfected with GD or isolated supernatant DNA from SN-38-treated HCT-116 (HCT-DNA) with (B) or without (C) LPS priming. HCT-116 cells were treated with SN-38 (500 nM) for $72 \mathrm{~h}$ before harvest of supernatant. (D) Immunoblot analysis of caspase 1 in LPS-primed BMMs transfected with $1.0 \mu \mathrm{g} / \mathrm{ml}$ of indicated DNA. Ile-DNA, DNA isolated from the ileum of CPT-11-treated mice. C57BL/6 mice were administrated with CPT-11 (90 mg/kg, i.p.) daily for 4 consecutive days and were sacrificed on day 5 for the harvest of ileum. $\beta$-actin served as a loading control. (E-I) Production of IL-1 $\beta$, IL-18, or IL-6 by BMMs (E-G) or BMDCs (H-I) from wildtype and $\mathrm{AIM}^{-/-}$mice. BMMs and BMDCs were treated as in D. (J-K) Production of IL-1 $\beta(\mathbf{J})$ and IL-6 (K) by LPS-primed BMDCs transfected with $1.0 \mu \mathrm{g} / \mathrm{ml}$ of HCT-DNA pre-treated with DNase or RNase. The data are representative of three independent experiments and depict means \pm SEM; ${ }^{*} P<0.05,{ }^{* *} P<0.01$, ${ }^{* * *} P<0.001$. GD, genomic DNA; NS, not significant; Pro-Casp1, procaspase 1; p10, active form of caspase 1.

(Figure 3F).

Previous studies reported that both ssDNA and dsDNA could be carried by exosomes [45-48]. To further characterise exosome DNA (exoDNA), we used S1 nuclease or dsDNase digestion. S1 nuclease degrades sin- gle-strand nucleic acids and dsDNase is a double-strand specific endonuclease. We observed a strong reduction of total extracted DNA in the dsDNase-treated samples, compared to those undigested or treated with S1 nuclease (Supplementary information, Figure S3B), demonstrating 
A

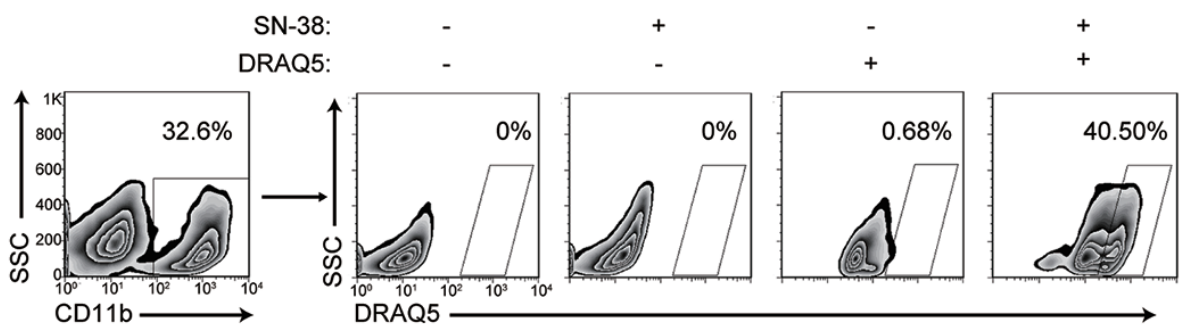

B
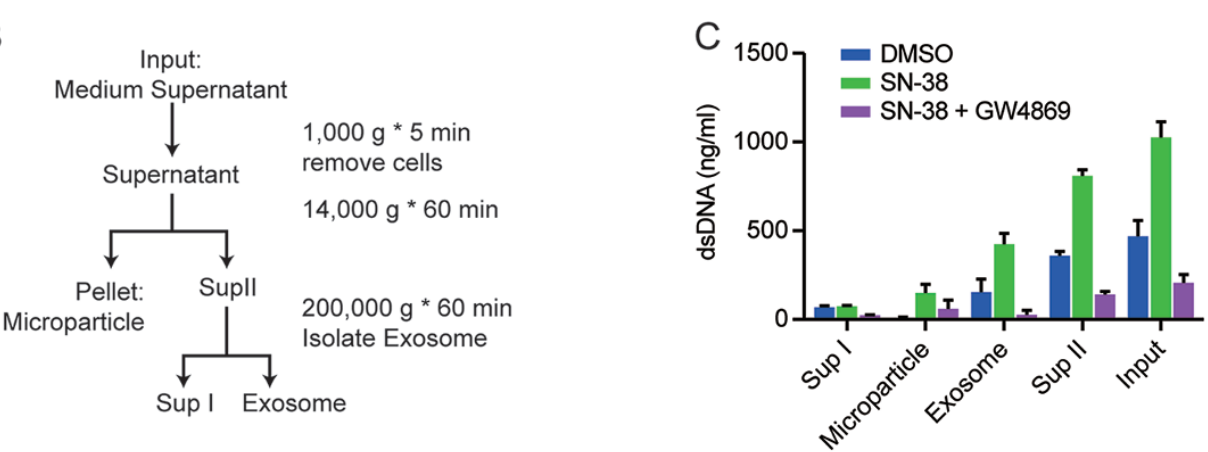

D
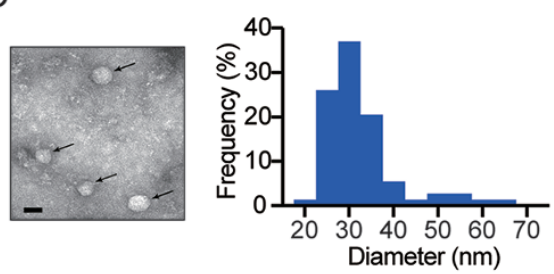

E
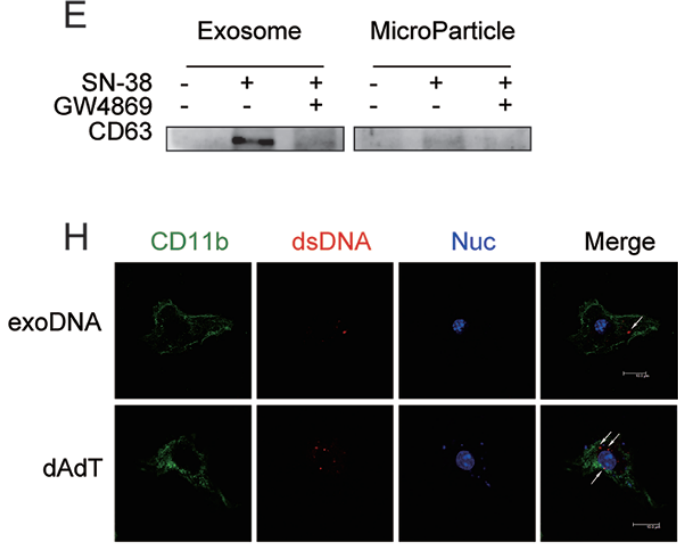
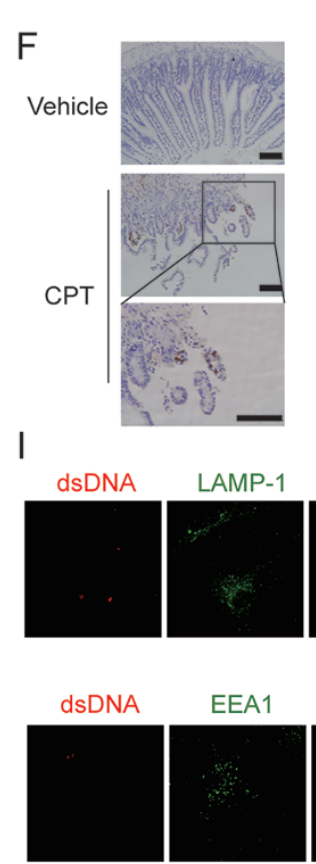

G
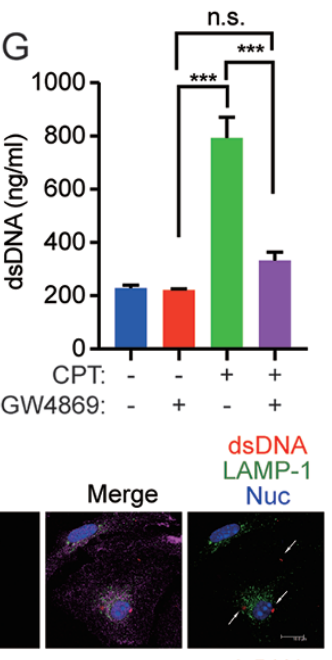

dsDNA
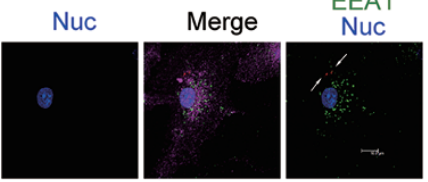

Figure 3 Released dsDNA can be transferred into macrophages via exosomes. (A) dsDNA entry into BMMs. HCT-116 cells pre-treated with SN38 (500 nM, $72 \mathrm{~h})$ were stained with DRAQ5 $(10 \mu \mathrm{M})$ and then co-cultured with BMMs for $24 \mathrm{~h}$ more. DRAQ5 staining in BMMs was analysed using flow cytometry. (B-E) SN-38-stimulated exosome release in HCT-116 cells. Cells were treated with indicated agents (SN-38, $500 \mathrm{nM}$; GW4869, $20 \mu \mathrm{M}$ ) for $72 \mathrm{~h}$ before harvesting the culture medium. (B) Flow chart of culture medium dissection; (C) concentrations of dsDNA in each fraction; (D) representative transmission electron microscope image (left panel) and size distribution (right panel) based on transmission electron microscope image analysis of exosome fractions. Scale bar, $25 \mathrm{~nm}$; (E) immunoblot analysis of CD63 in exosome fractions. The microparticle fraction was used as a control. (F) Immunohistochemistry staining of CD63 in ileum isolated from C57BL/6 mice treated with CPT11 (90 mg/kg, i.p.) for 4 consecutive days. Scale bar, $100 \mu \mathrm{m}$. (G) Concentrations of dsDNA in culture medium of HCT-116 cells. $(\mathbf{H}, \mathbf{I})$ Localisation of exosome dsDNA in BMMs. Mice BMMs were treated with isolated exosomes from SN-38 treated HCT-116 cells for 2-3 h, and DRAQ5-labelled poly(dA:dT) was transfected into BMMs with Lipofectamine 2000. dsDNA and subcellular organelles were stained using fluorescence confocal microscopy. (H) DRAQ5-labelled dsDNA (red), CD11b (green), and nuclei (DAPI, blue). (I) DRAQ5-labelled dsDNA (red), lysosomes (LAMP-1, upper, green), early endosomes (EEA1, lower, green), nuclei (blue), and $\beta$-actin (purple). The data are representative of three independent experiments and depict the means \pm SEM. 
that dsDNA was the dominant component in exoDNA. Moreover, exoDNA had a DNA origin similar to the input DNA (Supplementary information, Figure S3CS3D), suggesting exosome-mediated delivery of dsDNA. In agreement with this result, inhibition of exosome secretion by GW4869 largely attenuated dsDNA release stimulated by CPT-11, suggesting that dsDNA release was dependent on exosome secretion (Figure 3G). Collectively, these results suggested that dsDNA was secreted from intestinal epithelial cells via the exosome into the intercellular microenvironment.

We next examined whether exosomes containing the released dsDNA could be delivered into the cytosol. To this end, BMMs were incubated in culture medium containing pre-labelled exosomes isolated from SN-38treated HCT-116 cells. DNA packed in the exosomes was stained with DRAQ5. Indeed, DRAQ5-dsDNA in exosomes was also detected in the cytosol of BMMs by confocal microscopy, in a similar manner to the positive control transfected with DRAQ5-labelled poly(dA:dT) (Figure $3 \mathrm{H}$ ). We also examined the intracellular localization of the delivered DRAQ5-positive dsDNA by staining macrophages with the lysosomal marker LAMP-1 or the early endosomal marker EEA1. In a parallel experiment we could observe no obvious co-localisation between cytosolic DRAQ5-dsDNA with other organelles, such as lysosomes or early endosomes, suggesting an exosome-specific delivery route of DRAQ5-dsDNA to the phagocytic compartment (Figure 3I). Together, these data demonstrate that CPT-11 induces the release of dsDNA that is directly delivered into the cytosol of phagocytes via exosomes.

Blockage of exosome release reverses the CPT-11-induced intestinal toxicity

We next examined the function of exoDNA (exosome-released DNA) in CPT-11-induced inflammation. BMMs and peritoneal macrophages were stimulated with exoDNA and IL-1 $\beta$ production was measured. The results showed that exoDNA induced the apparent production of IL-1 $\beta$, which was largely abolished in the AIM2 KO (Supplementary information, Figure S4AS4B). Isolated exosomes from HCT-116 cells were also directly incubated with ileums in culture. This resulted in enhanced IL- $1 \beta$ secretion in the culture medium of the wild-type ileum but not the AIM2 KO ileum (Supplementary information, Figure S4C).

To validate the functional link between exosome secretion and CPT-11-induced intestinal inflammation in vivo, GW4869 was used to block exosome release in mice. CPT-11 was intraperitoneally injected for 4 consecutive days into C57BL/6 mice and GW4869 treatment was started 1 day ahead (Figure 4A). As expected, CPT11 induced dsDNA release into both peritoneal lavage fluid and fluid flushed from the small intestine were largely reversed by GW4869 treatment (Figure 4B-4C). Consistently, GW4869 significantly reduced severity of diarrhoea (Figure 4D), body weight loss (Figure 4E), and intestine shortening (Figure 4F-4G) following CPT11 treatment. Further histopathological analysis of the ileum confirmed that GW4869 largely reversed mucosal damage (Figure 4H) and villus shortening (Figure 4I) induced by CPT- 11 treatment. Together, these data indicate that exosome release was required for CPT-11-caused intestinal damage.

\section{AIM2 deficiency attenuates CPT-11-induced intestinal toxicity}

The aforementioned results suggested an exosome-mediated dsDNA delivery route into phagocytes that may be sensed by the AIM2 inflammasome during chemotherapy. To further confirm the functional requirement of the AIM2 inflammasome in CPT-11-induced intestinal inflammation and diarrhoea, we compared CPT-11-induced intestinal inflammation and diarrhoea between wild-type mice and AIM2-deficient mice. CPT-11 was intraperitoneally injected for 4 consecutive days, and the severity of mucositis was assessed as mentioned above. After challenge by CPT-11, AIM2-deficient mice showed delayed morbidity and lower lethality compared with wildtype mice (Figure 5A). On day 5, CPT-11 induced severe diarrhoea in the wild-type group, whereas AIM2-deficient mice had significantly reduced severity of diarrhoea (Figure 5B). Decreased disease severity was also accompanied by a reduction of small intestine shortening (Figure 5C-5D), but not colon shortening, in AIM2-deficient mice (Supplementary information, Figure S5A). The ileum histopathology revealed that AIM2-deficient mice had ameliorated inflammatory cell infiltration and mucosal damage, including epithelial ulceration, submucosal oedema (Figure 5E), and villi shortening (Figure $5 \mathrm{~F}$ ), upon CPT-11 challenge. In contrast, there were no obvious changes in colon histopathology (Supplementary information, Figure S5B). These observations indicated that AIM2 exacerbates the intestinal mucositis induced by CPT-11.

Consistent with the protective phenotype of AIM2-deficient mice, CPT-11 induced massive IL- $1 \beta$ and IL18 accumulation in the peritoneal lavage fluid in wildtype mice (Figure 5G-5H) measured on day 7 following CPT-11 challenge. Moreover, no differences in dsDNA release were detected between wild-type and AIM2-deficient mice (Supplementary information, Figure S5C). In contrast, although CPT-11 also triggered IL-6 secre- 
tion, there were no differences between wild-type and AIM2-deficient mice (Figure 5I). Similarly, mature IL$1 \beta$ secretion was highly induced by CPT- 11 in ileum explants and markedly attenuated in AIM2-deficient mice (Figure 5J). To further confirm activation of the AIM2 inflammasome in vivo, activity of caspase 1 p10 in the intestine was determined by immunoblot analysis. Upon CPT-11 administration, AIM2-deficient mice displayed decreased active $\mathrm{p} 10$ in the ileum (Figure $5 \mathrm{~K}-5 \mathrm{~L}$ ) but not in the colon (Supplementary information, Figure S5D$\mathrm{S} 5 \mathrm{E})$. These data indicated that AIM2 modulates intesti- nal mucositis in a manner dependent on IL-1 $\beta$ and IL-18 production, suggesting that CPT-11-triggers self-DNAinduced AIM2 inflammasome activation.

Very recently, $\mathrm{Hu}$ et al. [49] reported that the AIM2 inflammasome in intestinal epithelial cells accounted for irradiation-induced gastrointestinal syndrome. To further confirm the involvement of AIM2 activation in immune cells, we generated chimeric mice by transplanting bone marrow cells from wild-type or AIM2-deficient donors into lethally irradiated C57BL/6 mice. Reconstitution efficiency was validated by flow cytometric assessment
A

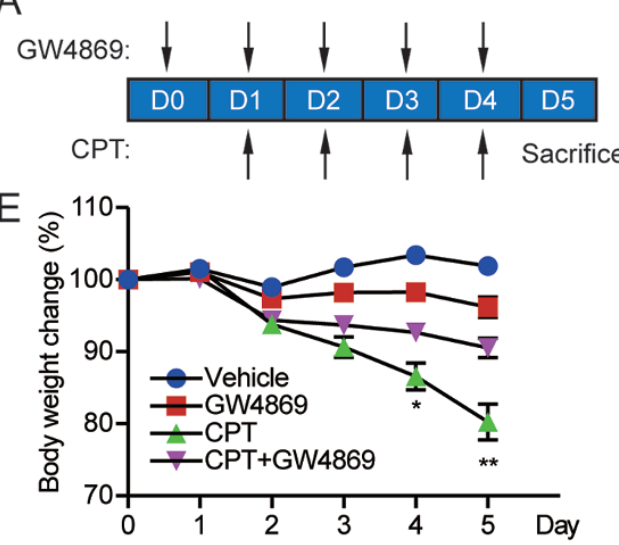

$\mathrm{F}$
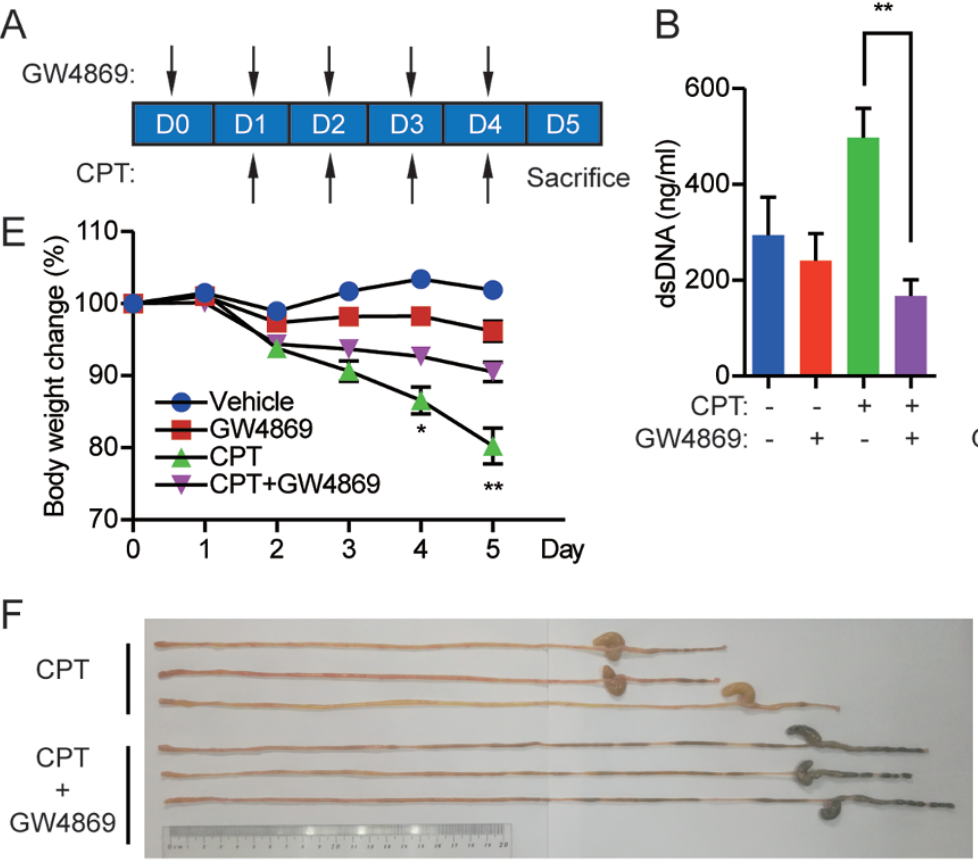

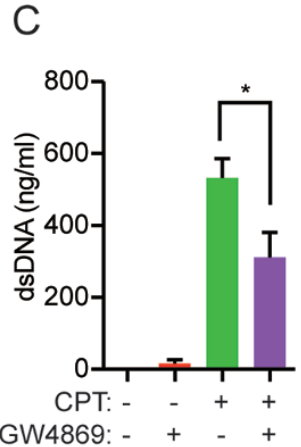

D

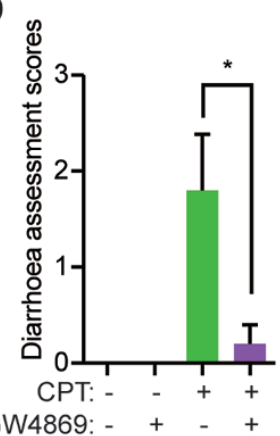

G

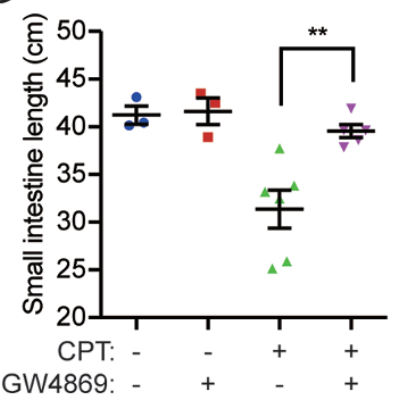

$\mathrm{H}$

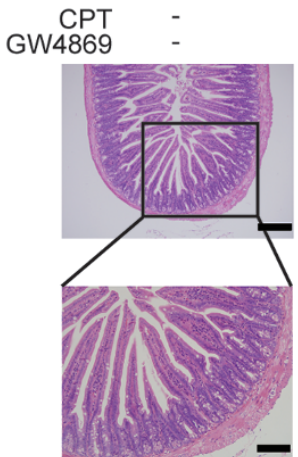

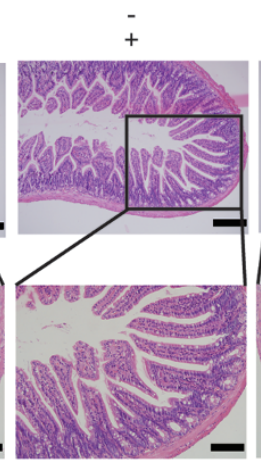
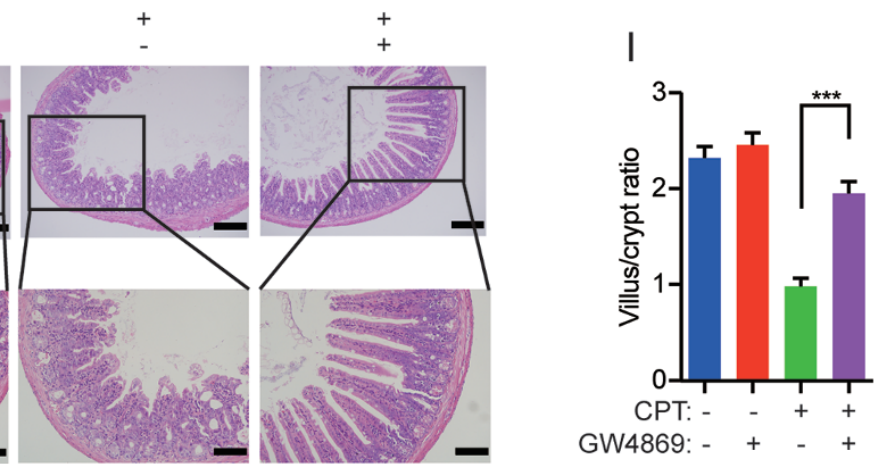

Figure 4 Blockage of exosome secretion reverses the CPT-11-induced intestinal toxicity. C57BL/6 mice were treated with CPT-11 (50 mg/kg, i.p.) daily for 4 consecutive days or combined with GW4869 (2.5 mg/kg, i.p.) from 1 day before CPT-11 administration until sacrifice. (A) Flow chart of drug administrations; (B) concentrations of dsDNA in cell-depleted fluid of a 3-cm length of ileum; (C) concentrations of dsDNA in cell-depleted peritoneal lavage fluid; (D) diarrhoea assessment scores; (E) body weight change; (F) images of small intestines; (G) length of small intestines; (H) representative images of H\&E staining of ileum sections; (I) villus/crypt ratio in H\&E-stained ileum sections. Scale bar, $200 \mu \mathrm{m}$ (upper) or $100 \mu \mathrm{m}$ (lower). 

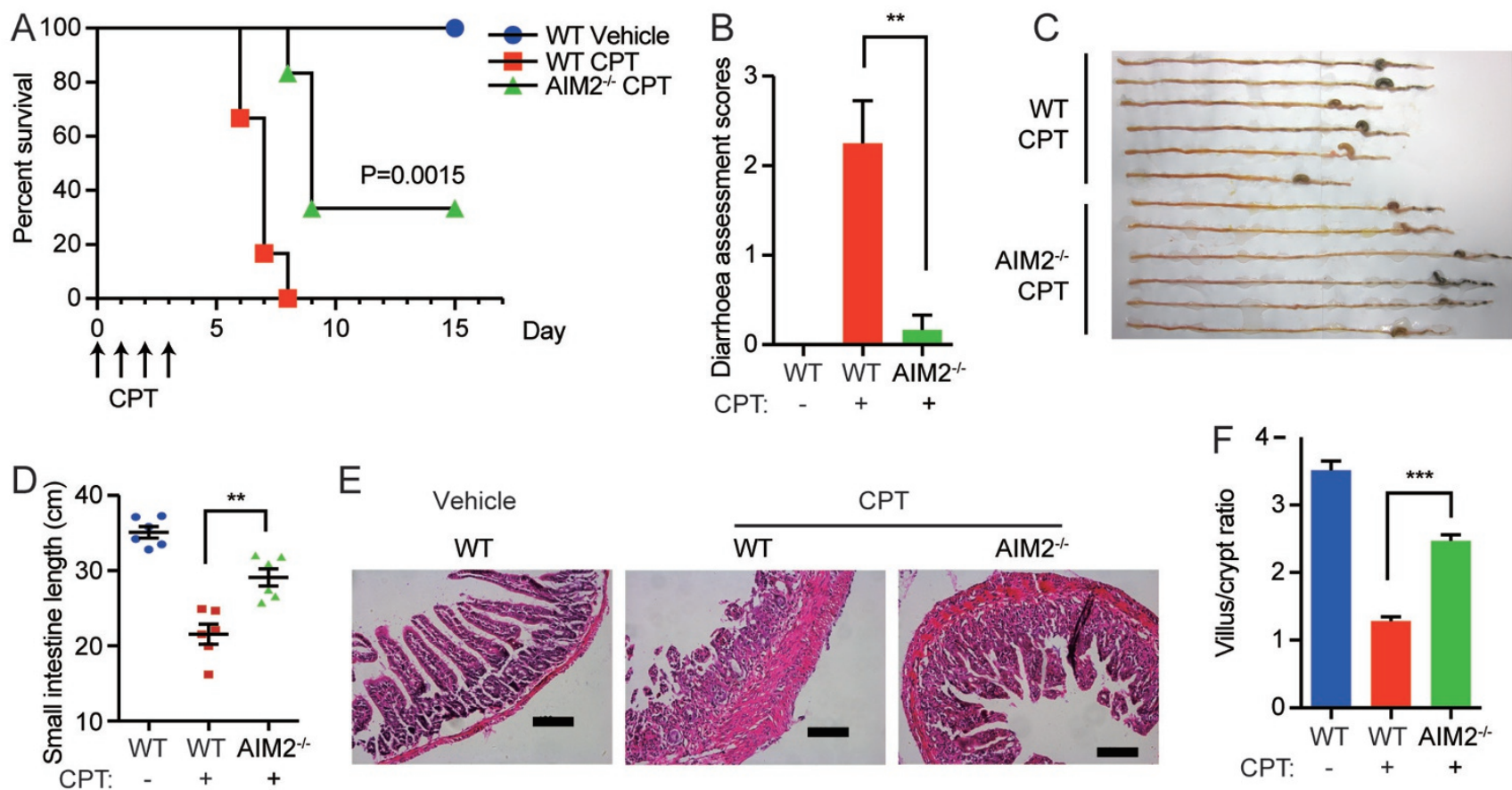

$E$
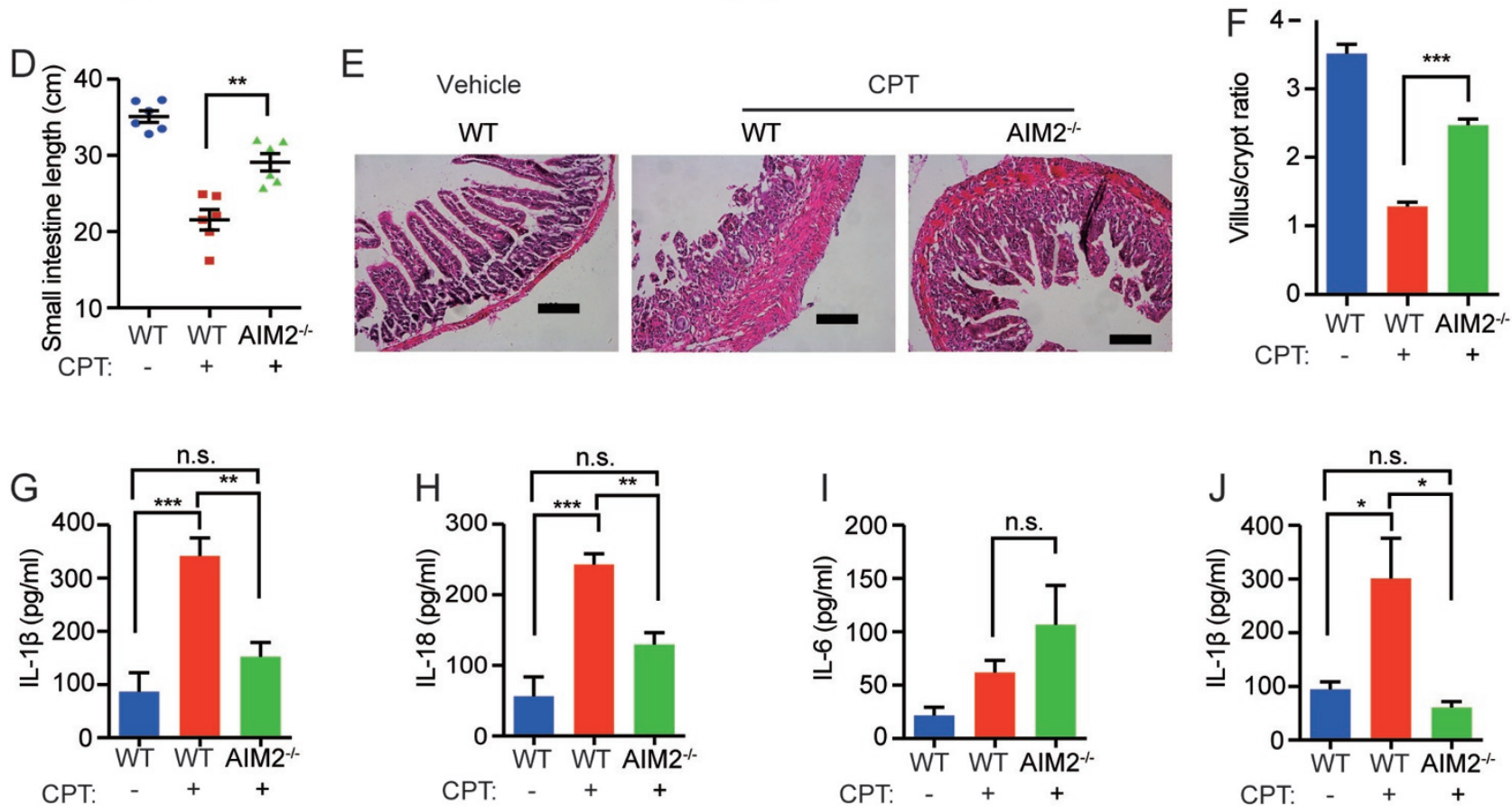

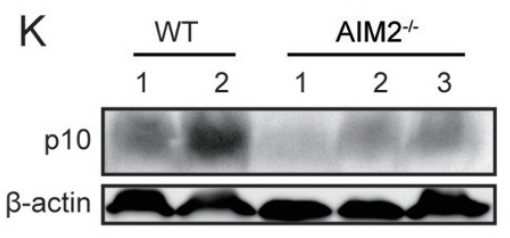

N

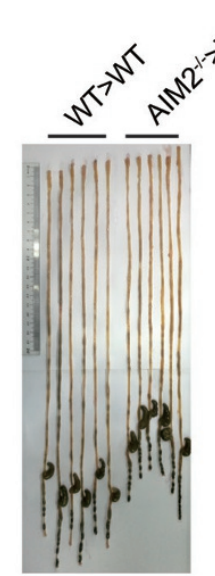

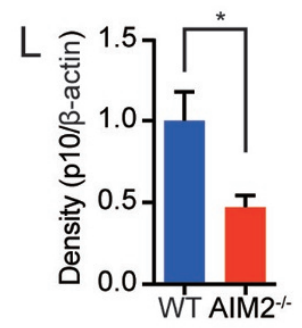

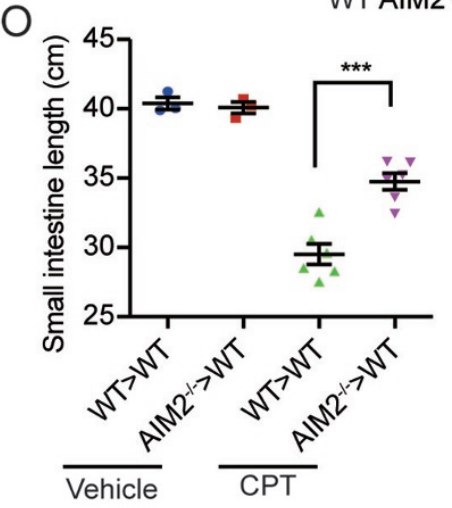

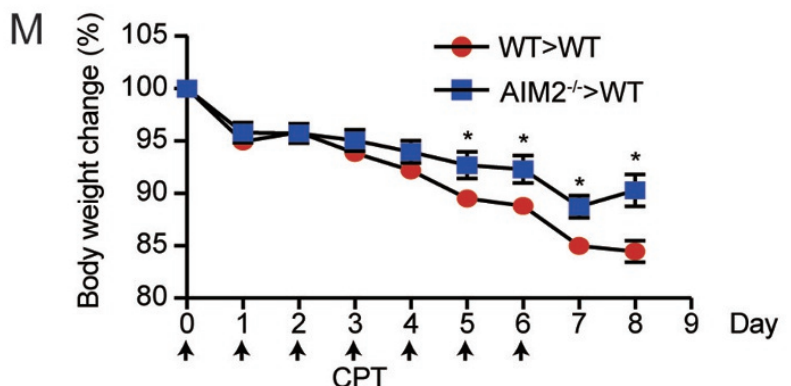

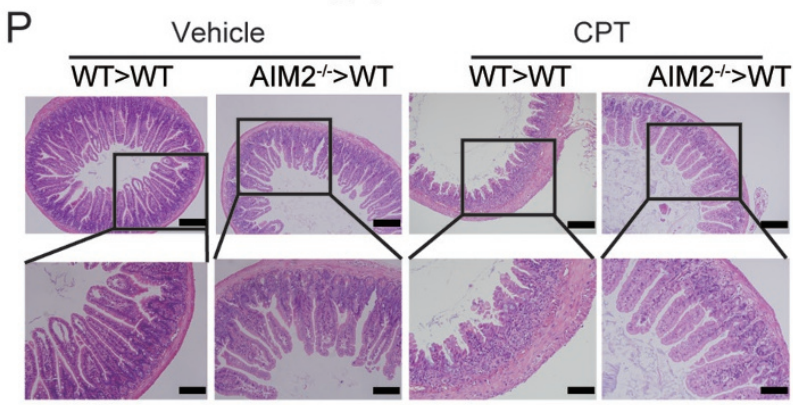


of the staining of CD45.1 and CD45.2 (at least 95\%, data not shown) and examining AIM2 expression levels in splenocytes and intestinal epithelial cells. AIM2 expression in splenocytes of wild-type mice reconstituted with AIM2-deficient bone marrow $\left(\mathrm{AIM}^{-/-}>\mathrm{WT}\right)$ was significantly depleted, compared with that in wild-type mice reconstituted with AIM2 wild-type bone marrow (WT $>$ WT). In the meanwhile, AIM2 expression in intestinal epithelial cells between AIM2 $2^{-/}>\mathrm{WT}$ and $\mathrm{WT}>\mathrm{WT}$ mice was comparable (Supplementary information, Figure S5F-S5G). Consistent with the phenotype of AIM2-deficient mice, AIM2 $2^{-/}>$WT mice showed less body weight loss (Figure $5 \mathrm{M}$ ) and small intestine shortening (Figure $5 \mathrm{~N}$ and 5O) than WT $>$ WT mice upon CPT11 challenge. Consistently, the ileum histopathological analysis revealed less intestinal damage in $\mathrm{AIM}^{-/}>\mathrm{WT}$ mice (Figure 5P). These data together highlight the role of AIM2 in innate immune cells in mediating CPT-11induced intestinal toxicity.

\section{AIM2 modulates CPT-11-induced intestinal mucositis in- dependently of the gut microbiota}

The mammalian intestine is colonized by large quantities of microorganisms, mainly bacteria, commonly referred to as the microbiota $[50,51]$. Microbial DNA is another ligand of the AIM2 inflammasome. Host-microbiota interactions can be mutually beneficial or deleterious, inciting intestinal inflammation [52]. To determine whether microbial DNA from the intestinal microbiota was indispensable for the effect of AIM2 deficiency on CPT-11-induced mucositis, we used a cocktail of antibiotics to remove the microbiota. The depletion efficiency was confirmed by assessing the bacterial 16S rDNA copy numbers in the faeces (Supplementary information, Figure S6A). Upon depletion of the microbiota, AIM2-deficient mice displayed remarkably reduced small intestine shortening following CPT-11 challenge (Supplementary information, Figure S6B). Histopathological analysis of the ileum revealed that the antibiotic-mediated microbiota depletion had no effect on the architecture of the intestine. Importantly, AIM2 deficiency protected mice against CPT-11-induced inflammatory cell infiltration, damage to the architecture of the submucosa, and ulceration in the absence of the gut microbiota (Supplementary information, Figure S6C). We next assessed the level of inflammasome activation in the absence of the microbiota. AIM2 deficiency was also associated with a significant decrease in the cleavage of active caspase 1 p10 (Supplementary information, Figure S6D-S6E). Collectively, these results suggest that microbial DNA is not associated with AIM2 inflammasome activation and that self-DNA occupies the dominant role during CPT-11 administration.

\section{Inhibition of the inflammasome alleviates CPT-11-in- duced intestinal toxicity}

Taken together, our results suggest that inhibition of the AIM2 inflammasome may provide a therapeutic solution for managing CPT-11-induced intestinal toxicity in the clinic. Despite a lack of specific inhibitors for this pathway, thalidomide, an immunomodulatory drug that inhibits inflammasome activation [53, 54], could be an option to attenuate dsDNA-mediated activation of the AIM2 inflammasome. In C57BL/6 mice, combination treatment with thalidomide reduced CPT-11-induced weight loss (Figure 6A) as well as intestine shortening (Figure 6B-6C). It also largely reversed histopathological damage (Figure 6D-6E), supporting its therapeutic potential for preventing CPT-11-induced intestinal toxicity. Importantly, thalidomide treatment did not reduce the anti-tumour effect of CPT-11. In the MC38 xenograft model, thalidomide rescued CPT-11-induced weight loss (Figure 6F) and histopathological damage (Figure 6J) without affecting efficacious tumour growth inhibition

Figure 5 AIM2-deficient mice exhibit attenuated intestinal mucositis and diarrhoea upon CPT treatment. (A-L) WT and $\mathrm{AIM}^{-1-}$ C57BL/6 mice were treated with CPT-11 (90 mg/kg, i.p) daily for 4 consecutive days and sacrificed on day 5 (B-J), 3 consecutive days and sacrificed on day 4 (K-L) or monitored daily for survival (A) ( $n=6$ per group). (A) Mice survival; (B) diarrhoea assessment scores; (C) images of small intestines; (D) length of small intestines; (E) representative images of H\&E staining of ileum sections. Scale bar, $100 \mu \mathrm{m}$; (F) villus length versus crypt depth ratio in H\&E-stained ileum sections; (G-I) production of IL-1 $\beta$, IL-18, and IL-6 in the peritoneal lavage fluid. One millilitre of PBS was injected into the peritoneal cavity and collected for cytokines analysis; (J) IL-1 $\beta$ production in explant fluid from 2-cm-long ileum pieces; (K) immunoblot analysis of caspase 1 p10 in ileum lysates treated with CPT-11 (90 mg/kg, i.p.) for 3 consecutive days; (L) densitometric quantification of the band intensity of $\mathrm{p} 10$ versus $\beta$-actin in (K). (M-P) AIM2 ${ }^{-/-}$chimera mice were constructed by transplanting bone marrow cells from WT $\left(\mathrm{WT}>\mathrm{WT}\right.$ ) or AIM2-deficient donor mice (AIM2 $\left.{ }^{-/}>\mathrm{WT}\right)$ into lethally irradiated C57BL/6 mice. Mice were then challenged with CPT-11 (50 mg/kg, i.p.) for 7 consecutive days and sacrificed on day 9. (M) Body weight changes; $(\mathrm{N})$ images of small intestines; $(\mathbf{O})$ length of small intestines; $(\mathbf{P})$ representative images of H\&E staining of ileum sections on day 9. Scale bar, $200 \mu \mathrm{m}$ (upper) or $100 \mu \mathrm{m}$ (lower). Each symbol represents one mouse (D, O). The data are representative of three independent experiments and depict the means \pm SEM. ${ }^{*} P<0.05$, ${ }^{* \star} P<0.01,{ }^{* * *} P<0.001$. NS, not significant; WT, wild-type. 
A

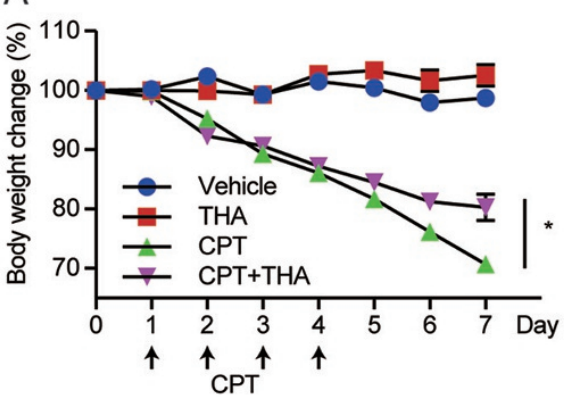

B

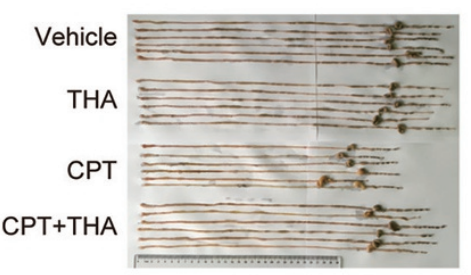

C

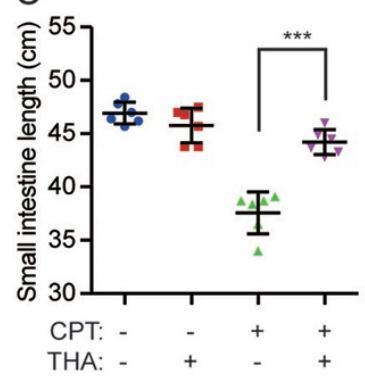

D CPT: THA:

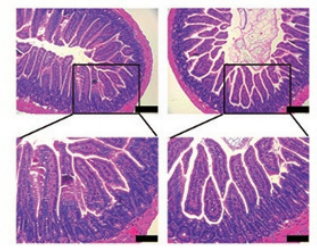

J

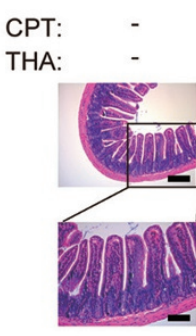

$+\quad+$

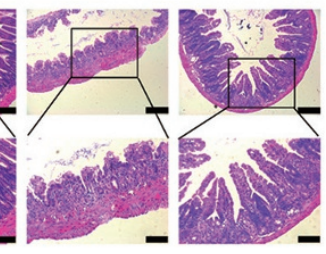

E

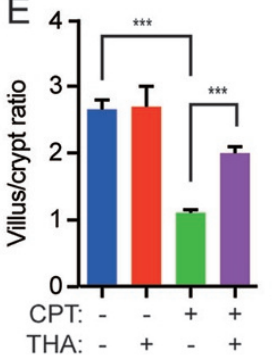

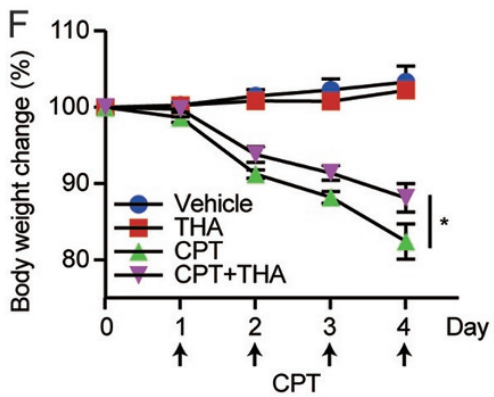
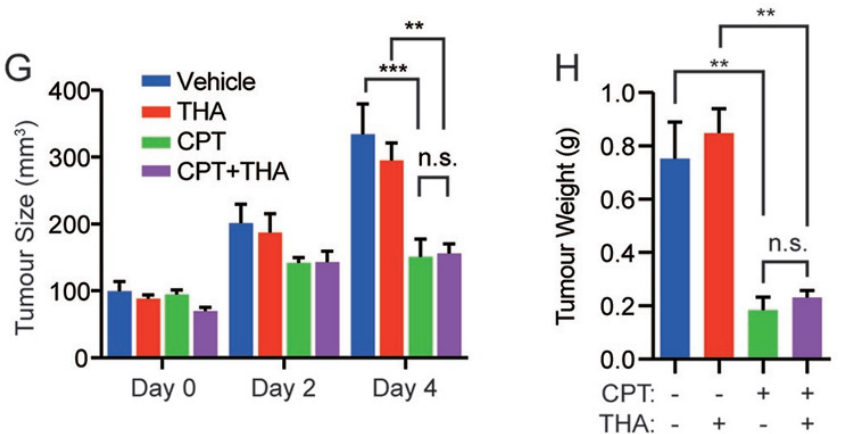

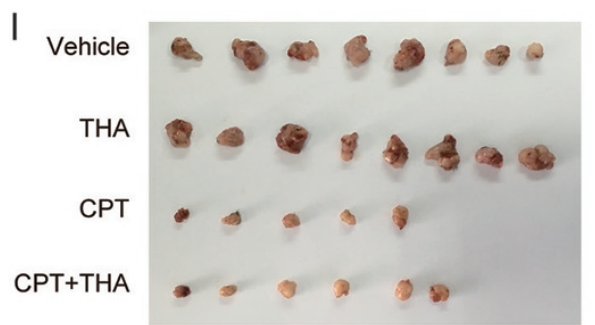

$\mathrm{K}$

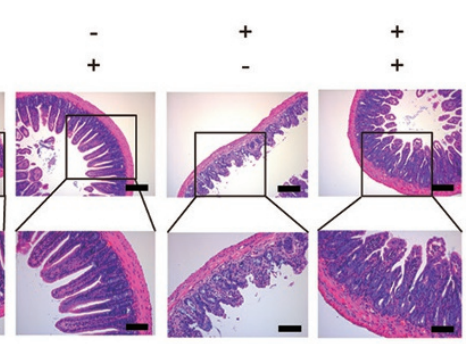

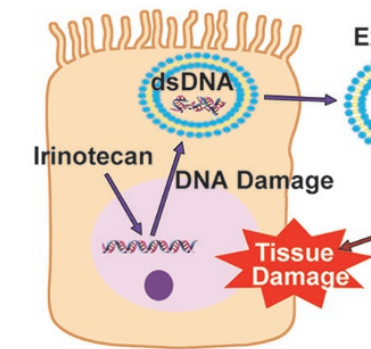

Intestinal Epithelial Cells

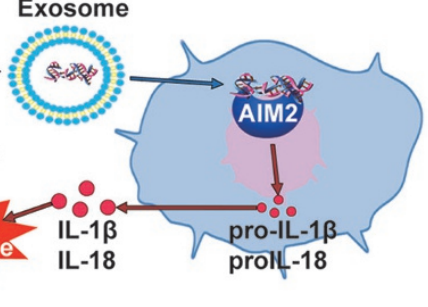

MФs \& DCs

Figure 6 Inhibition of the inflammasome ameliorates CPT-11-induced intestinal toxicity. (A-E) C57BL/6 mice were administered with CPT-11 (90 mg/kg, i.p.) for 4 consecutive days or thalidomide (THA, $100 \mathrm{mg} / \mathrm{kg}$, p.o.) daily from 1 day before CPT11 administration until sacrifice. On day 7 after the first administration, the mice were sacrificed, and the intestines were isolated for further analysis ( $n=6$ per group). (A) Body weight changes; (B) images of small intestines; (C) length of small intestines; (D) representative images of H\&E staining of ileum sections. Scale bar, $200 \mu \mathrm{m}$ (upper) or $100 \mu \mathrm{m}$ (lower); (E) analysis of the villus length versus crypt depth ratio in H\&E-stained ileum sections from (D). (F-J) C57BL/6 mice were injected with MC38 tumour cells to generate the tumour-bearing model. Ten days post injection, tumour-bearing mice were administered with CPT-11 (75 mg/kg, i.p.) for 4 consecutive days and THA (100 mg/kg, p.o.) daily from 1 day before CPT-11 administration until sacrifice. On day 5 after the first administration, the mice were sacrificed, and the intestines were isolated for further analysis. (F) Body weight changes; (G) tumour size; $(\mathbf{H})$ tumour weight on day 5; (I) tumour images on day 5; (J) representative images of H\&E-stained ileum sections. Scale bar, $100 \mu \mathrm{m}$. (K) Model of dsDNA-mediated intestinal toxicity via activation of the inflammasome during CPT-11 administration. The data are representative of three independent experiments and depict the means \pm SEM. ${ }^{*} P<0.05,{ }^{* *} P<0.01,{ }^{* * *} P<0.001$. NS, not significant. 
by CPT-11 treatment (Figure 6G-6I).

\section{Discussion}

Diarrhoea is a common side effect of cancer chemotherapy [55]. In many instances, diarrhoea can be a treatment-limiting or life-threatening side effect, resulting in dehydration, shock, renal impairment, and electrolyte disturbances [56]. Treatment with 5FU, oxaliplatin, capecitabine, and the alkylating agent cisplatin can also cause severe diarrhoea, but this side effect is less common with these treatments than with CPT-11 treatment [27].

In the present study, we found that, among a total of 69 advanced CRC patients, $\sim 40 \%$ exhibited grade 1-3 diarrhoea (Supplementary information, Figure S1A). The severity of the diarrhoea showed a close association with higher dsDNA production. We also found that CPT-11 directly triggered intestinal epithelial cells to release dsDNA in an exosome-mediated manner. CPT-11-induced dsDNA release from normal intestinal cells activated the AIM2 inflammasome in innate immune cells, accounting for the excessive intestinal inflammatory response following this treatment (Figure 6K). The exceptionally high incidence of CPT-11 may have resulted from the high concentration of active CPT-11 maintained in the intestinal microenvironment and was associated with excessive dsDNA exposure in the mouse model (Figure 4). In our study, we also found that dsDNA release was not restricted to CPT-11, and was also induced by another chemotherapeutic agent cisplatin (Supplementary information, Figure S1D). However, whether other chemotherapeutic-induced diarrhoea events are associated with release of self-dsDNA should be further explored.

AIM2 is commonly known as a DNA sensor that assembles and is part of the inflammasome complex. However, AIM2 mutations are frequently identified in CRC patients. Recently, two studies highlighted the role of AIM2 in modulating colorectal tumourigenesis via distinct mechanisms, both of which were inflammasome-independent. Wilson et al. [57] revealed that AIM2 suppressed colon tumourigenesis by inhibiting DNA-PK-mediated AKT activation, whereas another study indicated that AIM2 regulated stem cell proliferation in the intestinal mucosa [58]. Our results advance the current understanding of the role of AIM2 in CRC by revealing an inflammasome-dependent role that exacerbates chemotherapy-induced intestinal mucositis and diarrhoea. Of note, a very recent study reported that the AIM2 inflammasome was also essentially required in intestinal epithelial cells for irradiation-induced gastrointestinal syndrome. The mechanistic insights pointed to the role of nuclear AIM2, which is believed to sense radiation-induced DNA damage in the nucleus and mediates inflammasome activation and cell death [49]. These two studies complemented each other in advancing an understanding of the essential role of AIM2 in radiation and chemotherapy in relation to intestinal toxicity.

dsDNA recognition by different sensors has been extensively studied, yet a mechanistic link explaining the access of tumour cell-derived dsDNA relative to immune cells is lacking. Tumour-derived immune cell activation is mediated by soluble factors as well as by extracellular vesicles known as exosomes and microparticles [42]. In the present study, we demonstrated that CPT-11 induced rapidly proliferating cells, including tumour cells and intestinal epithelial cells, to release host DNA in an exosome-dependent manner. Exosomes are endosome-derived organelles with a diameter of 10-100 nm that contain proteins, lipids, RNA, DNA, and other molecules [39]. Like microparticles, exosomes mediate intercellular communication between tumour and immune cells and regulate immune signalling [41]. Exosomes function as "drivers" to deliver self-DNA into the cytosol of macrophages and DCs. These findings solve the unanswered question of how free DNA traverses the cell membrane to gain access to targeting sensors.

Treatment with CPT-11 can disrupt the intestinal mucosal barrier with increased epithelial apoptosis [27]. Rapid initiation of excessive epithelial apoptosis disrupts the barrier architecture and causes subsequent gut microbial translocation. This may be an important mediator in CPT-11-induced intestinal mucositis, as in dextran sulphate sodium-mediated colitis, which is induced mainly by translocation of gut bacteria. However, under conditions of gut microbiota depletion, a previous study [59] and our data (Supplementary information, Figure S6) revealed that intestinal damage and inflammatory cell infiltration also occurred upon CPT-11 challenge. These data suggest that the microbiota in the intestine were not the essential mediators and that excessive apoptosis may trigger intestinal damage via release of immunogenic mediators.

The immunogenicity of apoptotic cell death is controversial. In general, physiological cell death (apoptosis) is thought to be intrinsically tolerogenic, whereas pathological cell death (necrosis) is inherently immunogenic [60]. However, in-depth investigations have revealed that apoptotic cells can also be highly immunogenic, whereas necrotic cells are less immunogenic than cells undergoing an immunogenic form of apoptosis [61, 62]. Chemotherapy-induced cell death occurs frequently through apoptosis, including irinotecan administration [63]. However, whether self-DNA from chemotherapy-mediat- 
ed apoptosis provides a dangerous or a friendly signal to the host has not been fully characterized. Here, we report that self-dsDNA released by irinotecan induction can, in part, activate the AIM2 inflammasome, which may provide new insights into the immunogenicity of chemotherapy-induced apoptosis.

In addition to mechanistic insight, this study has identified a potentially valuable potential therapeutic application by providing a mechanism-based rationale for combating diarrhoea. The concurrent inhibition of AIM2 by an inflammasome inhibitor, thalidomide, alleviated CPT-11-induced intestinal toxicity without compromising its anticancer efficacy. Because thalidomide is clinically available for the treatment of multiple myeloma or solid tumours, our findings may be readily translated into a therapeutic opportunity that could be rapidly tested in clinical trials. Moreover, the exploration of therapeutic opportunities for thalidomide together with other compounds could also be promising.

\section{Materials and Methods}

\section{Patients}

Patients with CRC who attended Shanghai General Hospital were included in the present study with approval from the Institutional Review Board and informed consent from patients. All patients received irinotecan-contained chemotherapy (irinotecan alone or FOLFIRI). Peripheral blood was collected before and $48 \mathrm{~h}$ after treatment. The present study was approved by the Ethics Committee of Shanghai General Hospital.

\section{Mice}

AIM2-deficient mice were obtained from the Jackson Laboratory. C57BL/6 wild-type mice were purchased from the Shanghai Laboratory Animal Center. All mice were bred and maintained in specific pathogen-free conditions. Age (6-8 weeks) and sexmatched mice were used in the experiments. All animal experiments were undertaken in accordance with the NIH Guide for the Care and Use of Laboratory Animals with the approval of the Institutional Animal Care and Use Committee of Shanghai Institute of Materia Medica, the Institutional Animal Care and Use Committee of the Shanghai Institute of Biochemistry and Cell Biology, Chinese Academy of Sciences.

\section{Quantification of dsDNA}

Peritoneal lavage fluid was harvested using $1 \mathrm{ml}$ PBS. Fluid from $3-\mathrm{cm}$ lengths of intestine was harvested using $0.5 \mathrm{ml}$ PBS. Fluid and cell culture medium were subsequently centrifuged at $5000 \mathrm{rpm}$ for $5 \mathrm{~min}$, and the supernatants were centrifuged again. The second supernatants correspond to cell-depleted samples. dsDNA in the supernatants was quantitated using the PicoGreen assay (Life Technologies).

For patient serum samples, the whole blood of patients was collected at indicated time points post CPT-11 administration. Serum was collected following centrifugation of the blood samples, aliquoted and stored at $-80^{\circ} \mathrm{C}$. Prior to analysis, serum was thawed and diluted with $\mathrm{ddH}_{2} \mathrm{O}$, and mixed 1:1 with the dye PicoGreen (Life Technologies) diluted 1: 200 in TE buffer in a 96-well plate. Fluorescence was measured following excitation at $485 \mathrm{~nm}$ and emission wavelength at $528 \mathrm{~nm}$. DNA concentrations in serum were calculated by comparison to a standard curve from double-stranded DNA provided in the assay kit.

\section{Cytokine measurements}

The cytokines in the cell culture medium and in vivo cell-depleted fluid were measured by ELISA. The IL-1 $\beta$ and IL-6 ELISA kits were obtained from R\&D systems. The IL-18 ELISA kit was obtained from MBL International Corporation.

\section{Exosome isolation}

HCT-116 cells were cultured for $72 \mathrm{~h}$, and the culture supernatants collected and centrifuged at $1000 \times g$ for 5 min and $14000 \times$ $g$ for $60 \mathrm{~min}$. Exosomes were then harvested by centrifugation at $200000 \times g$ for $60 \min [42,43]$. The exosome pellet was suspended in $100 \mu \mathrm{l}$ PBS.

\section{Exosome DNA extraction and analysis}

ExoDNA was analyzed as previously reported [47, 48]. Briefly, exoDNA was extracted using the DNeasy Blood and Tissue Kit (Qiagen) according to the manufacturer's instructions. Finally, the DNA was eluted in $50 \mu 1$ of $\mathrm{AE}$ buffer and stored at $-20^{\circ} \mathrm{C}$ before further processing. For analysis, exoDNA was digested with $\mathrm{S} 1$ nuclease (Thermo Fisher) and dsDNase (Thermo Fisher) separately and subjected to electrophoresis on agarose gels. A plasmid (4 $\mathrm{kb})$ and a synthesized oligonucleotide ( $86 \mathrm{bp}$ ) were used as positive control of dsDNA and ssDNA, respectively.

\section{Confocal microscopy}

BMMs were plated overnight on coverslips. Isolated dsDNA-containing exosomes and poly(dA:dT) were labelled with 1 $\mu \mathrm{M}$ DRAQ5 (Invitrogen) for $30 \mathrm{~min}$ at room temperature and then added to the cell culture medium to achieve a $1 \mu \mathrm{g} / \mathrm{ml}$ final concentration of dsDNA. Two hours later, the cells were fixed with 4\% paraformaldehyde in PBS; permeabilized with Triton X-100; blocked with $10 \%$ FBS in PBS; and stained with anti-CD11b or anti- $\beta$-actin, anti-LAMP-1, and anti-EEA1. Nuclei were stained with DAPI. Confocal microscopy was performed using a Leica TCS SP2 instrument.

\section{Flow cytometry}

HCT-116 cells were treated with $500 \mathrm{nM} \mathrm{SN}-38$ for $24 \mathrm{~h}$ and then labelled with $10 \mu \mathrm{M}$ DRAQ5. After labelling, the HCT-116 cells were washed three times with PBS, followed by co-culture with untreated macrophages. Twenty-four hours later, cells were harvested and stained with anti-CD11b. Data were acquired with an LSR II (BD Biosciences) and analysed using FlowJo analytical software.

\section{Primary cells and DNA stimulation}

BMMs and dendritic cells were prepared as follows: bone marrow cells were flushed from the femurs and tibias of C57BL/6 mice and subsequently depleted of red blood cells using ammonium chloride. For BMMs, cells were seeded at a density of $1.5 \times$ $10^{6}$ cells per well in 24-well plates in DMEM supplemented with $20 \mathrm{ng} / \mathrm{ml}$ murine M-CSF. Fresh medium was added every 2 days. 
On day 5, cells were harvested. For BMDCs, cells were seeded at a density of $1 \times 10^{6}$ cells per well in 24-well plates in RPMI-1640 medium supplemented with $20 \mathrm{ng} / \mathrm{ml}$ GM-CSF. Fresh medium was added every 2 days. On day 7, cells were collected for analysis.

DNA was purified from the supernatant of SN-38-treated HCT116 cells or the fluid from CPT-11-treated mice using phenol/chloroform/isoamyl alcohol. The primary cells were primed with 200 $\mathrm{ng} / \mathrm{ml}$ LPS from Escherichia coli 0111:B4 (Sigma) for $3 \mathrm{~h}$ before transfection with DNA at the indicated concentration for $4 \mathrm{~h}$ using Lipofectamine 2000 (Invitrogen).

\section{$16 S$ rDNA copies}

For antibiotic treatment, $1 \mathrm{~g} / 1$ ampicillin sodium salt, $1 \mathrm{~g} / 1$ metronidazole, $0.5 \mathrm{~g} / 1$ vancomycin hydrochloride, and $1 \mathrm{~g} / \mathrm{l}$ of neomycin were added to the drinking water. After 2 weeks of treatment, mouse faeces were collected for phenol/chloroform/isoamyl alcohol bacterial genomic DNA purification. After centrifugation, the supernatant was concentrated with 0.8 volumes of isopropanol, washed twice with $75 \%$ ethanol, and resuspended in nuclease-free water.

The abundance of specific intestinal bacterial groups was measured by qPCR. Primer sequences for total bacterial and specific bacterial 16S rDNA genes as well as PCR conditions have been described previously [64]. qPCR analysis was determined as described previously [64]. Samples that were negative after 40 cycles were considered to indicate that $16 \mathrm{~S}$ rRNA was "not detected" (ND).

\section{CPT-11 treatment of mice}

Mice were treated with CPT-11 by intraperitoneal (i.p.) administration (Meilunbio) at a dose of $90 \mathrm{mg} / \mathrm{kg}$ for 4 consecutive days. The mice were sacrificed on day 5 for intestinal length measurement, histopathology of the intestine, and measurement of cytokines. The survival rate of the mice was monitored every day for up to 15 days. Diarrhoea severity was scored on day 5 as follows [25]: 0, normal (normal stool or absent); 1, slight (slightly wet and soft stool); 2, moderate (wet, unformed stool with perianal staining of the coat); and 3 , severe (watery stool with perianal staining of the coat).

In the in vivo exosome inhibitor experiment, mice were first treated with $2.5 \mathrm{mg} / \mathrm{kg} \mathrm{GW} 4869$ i.p. starting from day 0 to day 4, then treated with $50 \mathrm{mg} / \mathrm{kg}$ CPT-11 i.p. starting from day 1 to day 4 . At day 5, mice were sacrificed and analysed.

In the experiment to generate bone marrow chimeras, donor mice were from 8-10-week-old wild-type or AIM2-deficient mice and recipient mice were 8 -week-old wild-type mice. Recipient mice were lethally irradiated (8.5 Gy) and intravenously injected with $7.5 \times 10^{6}$ bone marrow cells isolated from donor mice $12 \mathrm{~h}$ post irradiation. After 8 -week bone marrow reconstitution, mice were treated with $50 \mathrm{mg} / \mathrm{kg} \mathrm{CPT}-11$ for 7 consecutive days. Bone marrow reconstitution efficiency was verified after 6 weeks by flow staining of CD45.1 and CD45.2 in blood leukocytes, which was at least $95 \%$.

\section{Tumour growth and treatments}

To generate tumour-bearing mice, MC38 tumour cells $(2 \times$ $\left.10^{6}\right)$ were subcutaneously injected into the flank of mice. CPT$11(75 \mathrm{mg} / \mathrm{kg}$ ) was administered (i.p.) for 4 consecutive days. The mice were treated orally with THA $(100 \mathrm{mg} / \mathrm{kg})$ daily for 5 days, starting 1 day before the first CPT-11 injection. On day 5, the mice were sacrificed by cervical dislocation.

\section{Statistical analysis}

The significance of mean values between two groups was analysed by a two-tailed Student's $t$-test. For significance analysis of the clinical study on patients, a paired $t$-test was used in the data presented in Figure 1A-1B and an unpaired $t$-test was used in the data presented in Figure 1C. Kaplan-Meier survival curves were tested using the log-rank (Mantel-Cox) test. Two-way ANOVA was used to assess the significance of changes in body weight. All statistical analyses were performed using GraphPad Prism software. Samples that were $>3$ s.d. from the mean were excluded as outliers. The data are presented as the means \pm SEM. For all tests, values of $P<0.05$ were considered statistically significant.

\section{Acknowledgments}

We thank Wangpeng Gu and Wenjuan Wang for the help with intravenous injection. We are very grateful to Prof YangXin Fu (University of Texas Southwestern Medical Center) for constructive suggestions to the study. This work was supported by "Personalized Medicines — Molecular Signature-based Drug Discovery and Development", Strategic Priority Research Program of the Chinese Academy of Sciences (XDA12020102), the Ministry of Science and Technology of China (2013CB530504, 2016YFA0502202, 2016YFA0502204), the Strategic Priority Research Program of the Chinese Academy of Sciences (XDB19000000), the National Natural Science Foundation of China (81361120409), and the National Science and Technology Major Project (2015ZX09101009).

\section{Author Contributions}

QL, JX and SY performed the experiments and analyzed the data; $\mathrm{MH}, \mathrm{JD}, \mathrm{BS}$ and $\mathrm{MG}$ conceived the project and provided supervision; HD collected patients serum samples; XS and AB contributed to animal experiments; QL, JX and MH wrote the manuscript. All the authors provided comments and suggestions to the manuscript.

\section{Competing Financial Interests}

The authors declare no competing financial interests.

\section{References}

1 Bracci L, Schiavoni G, Sistigu A, Belardelli F. Immune-based mechanisms of cytotoxic chemotherapy: implications for the design of novel and rationale-based combined treatments against cancer. Cell Death Differ 2014; 21:15-25.

2 Tesniere A, Schlemmer F, Boige V, et al. Immunogenic death of colon cancer cells treated with oxaliplatin. Oncogene 2010; 29:482-491.

3 Michaud M, Martins I, Sukkurwala AQ, et al. Autophagy-dependent anticancer immune responses induced by chemotherapeutic agents in mice. Science 2011; 334:1573-1577.

4 Apetoh L, Ghiringhelli F, Tesniere A, et al. Toll-like receptor 4-dependent contribution of the immune system to anticancer chemotherapy and radiotherapy. Nat Med 2007; 13:1050- 
1059.

5 Matzinger P. Tolerance, danger, and the extended family. Annu Rev Immunol 1994; 12:991-1045.

6 Rock KL, Hearn A, Chen CJ, Shi Y. Natural endogenous adjuvants. Springer Semin Immunopathol 2005; 26:231-246.

7 Garg AD, Krysko DV, Vandenabeele P, Agostinis P. DAMPs and PDT-mediated photo-oxidative stress: exploring the unknown. Photochem Photobiol Sci 2011; 10:670-680.

8 Garg AD, Nowis D, Golab J, Vandenabeele P, Krysko DV, Agostinis P. Immunogenic cell death, DAMPs and anticancer therapeutics: an emerging amalgamation. Biochim Biophys Acta 2010; 1805:53-71.

9 Ahn J, Barber GN. Self-DNA, STING-dependent signaling and the origins of autoinflammatory disease. Curr Opin Immunol 2014; 31:121-126.

10 Ahn J, Gutman D, Saijo S, Barber GN. STING manifests self DNA-dependent inflammatory disease. Proc Natl Acad Sci USA 2012; 109:19386-19391.

11 Krysko DV, Garg AD, Kaczmarek A, Krysko O, Agostinis P, Vandenabeele P. Immunogenic cell death and DAMPs in cancer therapy. Nat Rev Cancer 2012; 12:860-875.

12 Zitvogel L, Galluzzi L, Smyth Mark J, Kroemer G. Mechanism of action of conventional and targeted anticancer therapies: reinstating immunosurveillance. Immunity 2013; 39:7488.

13 Deng L, Liang H, Xu M, et al. STING-dependent cytosolic DNA sensing promotes radiation-induced type I interferon-dependent antitumor immunity in immunogenic tumors. Immunity 2014; 41:843-852.

14 Woo S-R, Fuertes Mercedes B, Corrales L, et al. STING-dependent cytosolic DNA sensing mediates innate immune recognition of immunogenic tumors. Immunity 2014; 41:830842.

15 Ahn J, Xia T, Konno H, Konno K, Ruiz P, Barber GN. Inflammation-driven carcinogenesis is mediated through STING. Nat Commun 2014; 5:5166.

16 Bleiberg H, Cvitkovic E. Characterisation and clinical management of CPT-11 (irinotecan)-induced adverse events: the European perspective. Eur J Cancer 1996; 32A Suppl 3:S1823.

17 Merrouche Y, Extra JM, Abigerges D, et al. High dose-intensity of irinotecan administered every 3 weeks in advanced cancer patients: a feasibility study. J Clin Oncol 1997; 15:1080-1086.

18 Cunningham D, Pyrhönen S, James RD, et al. Randomised trial of irinotecan plus supportive care versus supportive care alone after fluorouracil failure for patients with metastatic colorectal cancer. Lancet 1998; 352:1413-1418.

19 Benson AB 3rd, Ajani JA, Catalano RB, et al. Recommended guidelines for the treatment of cancer treatment-induced diarrhea. J Clin Oncol 2004; 22:2918-2926.

20 Lima-Junior RC, Freitas HC, Wong DV, et al. Targeted inhibition of IL-18 attenuates irinotecan-induced intestinal mucositis in mice. Br J Pharmacol 2014; 171:2335-2350.

21 Xynos ID, Karadima ML, Voutsas IF, et al. Chemotherapy \pm cetuximab modulates peripheral immune responses in metastatic colorectal cancer. Oncology 2013; 84:273-283.

22 Tsavaris N, Voutsas IF, Kosmas C, Gritzapis AD, Baxevanis $\mathrm{CN}$. Combined treatment with bevacizumab and standard che- motherapy restores abnormal immune parameters in advanced colorectal cancer patients. Invest New Drugs 2012; 30:395402.

23 Melo ML, Brito GA, Soares RC, et al. Role of cytokines (TNF-alpha, IL-1beta and KC) in the pathogenesis of CPT11-induced intestinal mucositis in mice: effect of pentoxifylline and thalidomide. Cancer Chemother Pharmacol 2008; 61:775-784.

24 Arifa RD, Madeira MF, de Paula TP, et al. Inflammasome activation is reactive oxygen species dependent and mediates irinotecan-induced mucositis through IL-1beta and IL-18 in mice. Am J Pathol 2014; 184:2023-2034.

25 Guabiraba R, Besnard AG, Menezes GB, et al. IL-33 targeting attenuates intestinal mucositis and enhances effective tumor chemotherapy in mice. Mucosal Immunol 2014; 7:1079-1093.

26 Ahn J, Konno H, Barber GN. Diverse roles of STING-dependent signaling on the development of cancer. Oncogene 2015; 34:5302-5308.

$27 \mathrm{Hu}$ Z, Yang X, Ho PC, et al. St. John's Wort modulates the toxicities and pharmacokinetics of CPT-11 (irinotecan) in rats. Pharm Res 2005; 22:902-914.

28 Dambacher J, Beigel F, Seiderer J, et al. Interleukin 31 mediates MAP kinase and STAT1/3 activation in intestinal epithelial cells and its expression is upregulated in inflammatory bowel disease. Gut 2007; 56:1257-1265.

29 de Jong PR, Takahashi N, Harris AR, et al. Ion channel TRPV1-dependent activation of PTP1B suppresses EGFR-associated intestinal tumorigenesis. J Clin Invest 2014; 124:3793-3806.

30 Thurston TL, Ryzhakov G, Bloor S, von Muhlinen N, Randow F. The TBK1 adaptor and autophagy receptor NDP52 restricts the proliferation of ubiquitin-coated bacteria. Nat Immunol 2009; 10:1215-1221.

31 Yang S, Wang B, Humphries F, et al. Pellino3 ubiquitinates RIP2 and mediates Nod2-induced signaling and protective effects in colitis. Nat Immunol 2013; 14:927-936.

32 West AP, Khoury-Hanold W, Staron M, et al. Mitochondrial DNA stress primes the antiviral innate immune response. $\mathrm{Na}$ ture 2015; 520:553-557.

33 Hornung V, Ablasser A, Charrel-Dennis M, et al. AIM2 recognizes cytosolic dsDNA and forms a caspase-1-activating inflammasome with ASC. Nature 2009; 458:514-518.

34 Fernandes-Alnemri T, Yu JW, Datta P, Wu J, Alnemri ES. AIM2 activates the inflammasome and cell death in response to cytoplasmic DNA. Nature 2009; 458:509-513.

35 Burckstummer T, Baumann C, Bluml S, et al. An orthogonal proteomic-genomic screen identifies AIM2 as a cytoplasmic DNA sensor for the inflammasome. Nat Immunol 2009; 10:266-272.

36 Rathinam VA, Jiang Z, Waggoner SN, et al. The AIM2 inflammasome is essential for host defense against cytosolic bacteria and DNA viruses. Nat Immunol 2010; 11:395-402.

37 Fernandes-Alnemri T, Yu JW, Juliana C, et al. The AIM2 inflammasome is critical for innate immunity to Francisella tularensis. Nat Immunol 2010; 11:385-393.

38 Latz E, Xiao TS, Stutz A. Activation and regulation of the inflammasomes. Nat Rev Immunol 2013; 13:397-411.

39 Azmi AS, Bao B, Sarkar FH. Exosomes in cancer development, metastasis, and drug resistance: a comprehensive re- 
view. Cancer Metastasis Rev 2013; 32:623-642.

40 Valadi H, Ekstrom K, Bossios A, Sjostrand M, Lee JJ, Lotvall JO. Exosome-mediated transfer of mRNAs and microRNAs is a novel mechanism of genetic exchange between cells. Nat Cell Biol 2007; 9:654-659.

41 Valenti R, Huber V, Iero M, Filipazzi P, Parmiani G, Rivoltini L. Tumor-released microvesicles as vehicles of immunosuppression. Cancer Res 2007; 67:2912-2915.

42 Zhang H, Tang K, Zhang Y, et al. Cell-free tumor microparticle vaccines stimulate dendritic cells via cGAS/STING signaling. Cancer Immunol Res 2015; 3:196-205.

43 Mause SF, Weber C. Microparticles: protagonists of a novel communication network for intercellular information exchange. Circ Res 2010; 107:1047-1057.

44 Regev-Rudzki N, Wilson DW, Carvalho TG, et al. Cell-cell communication between malaria-infected red blood cells via exosome-like vesicles. Cell 2013; 153:1120-1133.

45 Balaj L, Lessard R, Dai L, et al. Tumour microvesicles contain retrotransposon elements and amplified oncogene sequences. Nat Commun 2011; 2:180.

46 Skog J, Wurdinger T, van Rijn S, et al. Glioblastoma microvesicles transport RNA and proteins that promote tumour growth and provide diagnostic biomarkers. Nat Cell Biol 2008; 10:1470-1476.

47 Thakur BK, Zhang H, Becker A, et al. Double-stranded DNA in exosomes: a novel biomarker in cancer detection. Cell Res 2014; 24:766-769.

48 Kahlert C, Melo SA, Protopopov A, et al. Identification of double-stranded genomic DNA spanning all chromosomes with mutated KRAS and p53 DNA in the serum exosomes of patients with pancreatic cancer. J Biol Chem 2014; 289:38693875.

$49 \mathrm{Hu} \mathrm{B}$, Jin $\mathrm{C}$, Li HB, et al. The DNA-sensing AIM2 inflammasome controls radiation-induced cell death and tissue injury. Science 2016; 354:765-768.

50 Perez-Lopez A, Behnsen J, Nuccio SP, Raffatellu M. Mucosal immunity to pathogenic intestinal bacteria. Nat Rev Immunol 2016; 16:135-148.
51 Kamada N, Seo SU, Chen GY, Nunez G. Role of the gut microbiota in immunity and inflammatory disease. Nat Rev Immunol 2013; 13:321-335.

52 Abraham C, Cho JH. Inflammatory bowel disease. $N$ Engl J Med 2009; 361:2066-2078.

53 Zitvogel L, Kepp O, Galluzzi L, Kroemer G. Inflammasomes in carcinogenesis and anticancer immune responses. Nat Immunol 2012; 13:343-351.

54 Keller M, Sollberger G, Beer HD. Thalidomide inhibits activation of caspase-1. J Immunol 2009; 183:5593-5599.

55 Carr C, Ng J, Wigmore T. The side effects of chemotherapeutic agents. Curr Anaesth Crit Care 2008; 19:70-79.

56 Sharma R, Tobin P, Clarke SJ. Management of chemotherapy-induced nausea, vomiting, oral mucositis, and diarrhoea. Lancet Oncol 2005; 6:93-102.

57 Wilson JE, Petrucelli AS, Chen L, et al. Inflammasome-independent role of AIM2 in suppressing colon tumorigenesis via DNA-PK and Akt. Nat Med 2015; 21:906-913.

58 Man SM, Zhu Q, Zhu L, et al. Critical role for the DNA sensor AIM2 in stem cell proliferation and cancer. Cell 2015; 162:45-58.

59 Pedroso SHSP, Vieira AT, Bastos RW, et al. Evaluation of mucositis induced by irinotecan after microbial colonization in germ-free mice. Microbiology 2015; 161:1950-1960.

60 Green DR, Ferguson T, Zitvogel L, Kroemer G. Immunogenic and tolerogenic cell death. Nat Rev Immunol 2009; 9:353-363.

61 Casares N, Pequignot MO, Tesniere A, et al. Caspase-dependent immunogenicity of doxorubicin-induced tumor cell death. J Exp Med 2005; 202:1691-1701.

62 Zitvogel L, Casares N, Péquignot MO, Chaput N, Albert ML, Kroemer G. Immune response against dying tumor cells. Adv Immunol 2004; 84:131-179.

63 Zitvogel L, Apetoh L, Ghiringhelli F, Kroemer G. Immunological aspects of cancer chemotherapy. Nat Rev Immunol 2008; 8:59-73.

64 Barman M, Unold D, Shifley K, et al. Enteric salmonellosis disrupts the microbial ecology of the murine gastrointestinal tract. Infect Immun 2008; 76:907-915.

(Supplementary information is linked to the online version of the paper on the Cell Research website.) 